\title{
The star formation history of luminous infrared galaxies
}

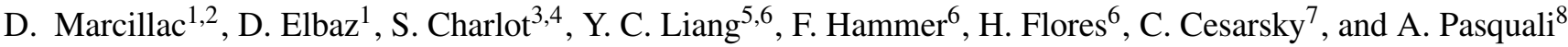 \\ 1 DSM/DAPNIA/Service d'Astrophysique, CEA/SACLAY, 91191 Gif-sur-Yvette Cedex, France \\ 2 Steward Observatory, University of Arizona, 933 N. Cherry Avenue, Tucson, AZ 85721, USA \\ e-mail: dmarci@as.arizona.edu \\ 3 Max-Planck-Institut für Astrophysik, Karl-Schwarzschild-Strasse 1, 85748 Garching, Germany \\ 4 Institut d'Astrophysique de Paris, CNRS, 98 bis boulevard Arago, 75014 Paris, France \\ e-mail: charlot@iap.fr \\ 5 National Astronomical Observatories, Chinese Academy of Sciences, No. 20A Datun Road, Chaoyang District, Beijing 100012, \\ PR China \\ e-mail: ycliang@bao.ac.cn \\ 6 GEPI, Observatoire de Paris, Section de Meudon, 92195 Meudon Cedex, France \\ e-mail: [hector.flores; francois.hammer]@obspm.fr \\ 7 ESO, Karl-Schwarzschild Strase 2, 85748 Garching bei Munchen, Germany \\ e-mail: ccesarsk@eso.org \\ 8 Max-Planck-Institut fuer Astronomie, Koenigstuhl 17, 69117 Heidelberg, Germany \\ e-mail: pasquali@phys.ethz.ch
}

Received 10 February 2006 / Accepted 26 May 2006

\begin{abstract}
Aims. We constrain the past star formation histories of a sample of 25 distant $(\bar{z} \sim 0.7)$ luminous infrared galaxies (LIRGs) detected with the mid infrared cameras ISOCAM and MIPS onboard the ISO and Spitzer satellites.

Methods. We used high-resolution VLT-FORS2 spectroscopy in addition to a comprehensive library of 200000 model optical spectra to derive Bayesian likelihood estimates of the star formation histories of these galaxies, based on analysis of Balmer absorption lines

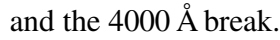

Results. The locus of distant LIRGs in the diagram defined by $\mathrm{H} \delta_{\mathrm{A}}$ and $\mathrm{D} 4000$ is roughly comparable to that of local LIRGs observed with IRAS, suggesting that no trend toward an evolution is detected between the local and distant LIRGs. We obtain similar results when using either the $\mathrm{H} 8$ or the $\mathrm{H} \delta_{\mathrm{A}}$ Balmer absorption-line indices in combination with $\mathrm{D} 4000$.

By computing a birthrate parameter $(b=S F R /\langle S F R\rangle)$ of $4 \pm 1$, we confirme that the distant LIRGs are currently experiencing a major phase of star formation. The most likely duration of the bursts is $0.10_{-0.06}^{+0.16} \mathrm{Gyr}$, during which the LIRGs produce $\sim 5-10 \%$ of their current stellar mass. No evidence was found for successive starbursts on the scale of a few times $10^{7} \mathrm{yr}$, such as those predicted by some numerical simulations of major mergers. However, the high number density of those galaxies suggests that they could have experienced between two and four LIRG phases until the present epoch. This scenario is not consistent with the formation of the $z \sim 0.7$ LIRGs through the continuous star formation characterizing isolated spiral galaxies as has been independently argued based on their morphology. Instead, minor mergers, tidal interactions, or gas accretion remain plausible triggering mechanisms for more than half of the distant LIRGs that do not harbor the morphology of major mergers.
\end{abstract}

Key words. galaxies: evolution - infrared: galaxies - galaxies: starburst

\section{Introduction}

It has been suggested that luminous infrared (IR) galaxies, i.e. galaxies radiating more than $\sim 90 \%$ of their light above $5 \mu \mathrm{m}$, have been to provide important constraints on galaxy formation and evolution. They are considered to be the main cause of the cosmic infrared background (CIRB) and major contributors to the evolution with redshift of the cosmic star formation rate (CSFR) of galaxies (Elbaz et al. 2002; Chary \& Elbaz 2001; see also the review by Lagache et al. 2005). The role of luminous IR galaxies (LIRGs for $10^{11} L_{\odot} \leq L_{\mathrm{IR}}=L[8-1000 \mu \mathrm{m}]<10^{12} L_{\odot}$ ) and ultra-luminous IR galaxies (ULIRGs for $L_{\mathrm{IR}} \geq 10^{12} L_{\odot}$ ) in the local universe could be neglected since they only produce about $6 \%$ of the integrated IR emission of local galaxies, hence $2 \%$ of their bolometric luminosity (Soifer \& Neugebauer 1991). But they rapidly evolve with redshift and dominate the CSFR above $z \sim 0.6$ (Le Floc'h et al. 2005). The IRAS extragalactic surveys have already suggested that their number density evolved rapidly with redshift $\left(\sim(1+z)^{7.6 \pm 3.2}\right.$ up to $z \sim 0.2$; Kim \& Sanders 1998). This rapid evolution was later confirmed up to $z \sim 1$ with ISOCAM and ISOPHOT onboard ISO at 15 and $170 \mu \mathrm{m}$ (see Elbaz et al. 2005, and references therein), above $z \sim 2$ using SCUBA at $850 \mu \mathrm{m}$ (Smail et al. 2001; Blain et al. 1999), and in the intermediate redshift range with MIPS onboard Spitzer at $24 \mu \mathrm{m}$ (Chary et al. 2004; Papovitch et al. 2004; Le Floc'h et al. 2005). However, little is known about the characteristics of the starbursts themselves, e.g. the amount of stars born during the burst, the burst duration, and even less about the physical processes responsible for the intense activity of these galaxies. One of the major reasons for this lack of information comes from their nature itself, i.e. the strong dust obscuration of their optical light.

In a previous paper (Liang et al. 2004, hereafter Paper I), we presented an analysis of the emission line properties of the galaxies. The star formation rates $(S F R)$, derived from the Balmer emission lines ( $\mathrm{H} \alpha$ and/or $\mathrm{H} \beta$, plus $\mathrm{H} \gamma$ to derive dust 
attenuations) were corrected for dust attenuation and found to be consistent with the ones derived from the mid IR (MIR) using the technique described in Chary \& Elbaz (2001). This study showed that LIRGs in general are not completely obscured by dust and that the use of high-resolution optical spectroscopy $(\Delta \lambda / \lambda=2000$, in the rest frame of the objects) could be used to derive intrinsic luminosities, hence $S F R$, in rough agreement with the IR-derived $S F R$, by minimizing the contamination by sky emission lines and allowing better corrections for underlying photospheric absorption lines. However, the consistent derivation of the signal-to-noise $(S / N)$ ratio on the intrinsic luminosities lead to large uncertainties on the measured visual attenuation. Moreover, there is evidence of some completely obscured star formation as found in the most luminous objects studied in Flores et al. (2004) or in Cardiel et al. (2003). The limited statistics of those studies clearly call for an extention of the sample of distant LIRGs, with good $S / N$ on the optical continuum and high spectral resolution, to robustly determine which fraction of the star formation taking place in LIRGs and ULIRGs is completely obscured by dust. However Hopkins et al. (2003) show that $S F R([\mathrm{OII}]), S F R(1.4 \mathrm{GHz})$, and $S F R(\mathrm{FIR})$ are in very good agreement for a larger sample of local infrared galaxies detected with IRAS and spectroscopically observed with the Sloan Digital Sky Survey (SDSS).

In the present paper, we wish to address the problem from another angle: stars less massive, hence with longer lifetimes, than those responsible for the emission lines standardly used to derive the optical $S F R$ of galaxies can escape their parent giant molecular cloud (GMC), their spectral signature might be used to derive key parameters concerning the starburst. The $\mathrm{H} \alpha$ light used to derive an $S F R$ is dominantly produced by the ionizing photons arising from stars more massive than $\sim 10 M_{\odot}$, with lifetimes shorter than 3 Myr. Those stars never escape their parent GMC (average lifetime of $10 \mathrm{Myr}$ ) and the dense regions of very strong extinction, in contrast to the $\mathrm{A}$ and $\mathrm{F}$ stars, which are the main contributors to the Balmer absorption lines and the $4000 \AA$ break.

We used these signatures of the optical continuum to compare distant LIRGs to nearby IRAS galaxies or synthetic spectra generated with the Bruzual \& Charlot (2003) model. After finding a signature of the starbursts in the $\mathrm{D} 4000-\mathrm{H} \delta$ diagram we used it to derive the burst properties.

Paper I indirectly confirmed the strong role played by LIRGs in the CSFR history with the derivation of gas metallicities in distant LIRGs twice lower that are the one measured in presentday galaxies of equal absolute $\mathrm{B}$ band magnitude. This result suggests that these galaxies have produced about half of their metals between $z \sim 1$ and today. It was also suggested that such a large metal production, as well as the large contribution of LIRGs to the CSFR and CIRB, could not result from a single burst phase in the galaxies harboring LIRG phases and hence that those galaxies must have experienced a series of LIRG phases in their lifetime. This possibility is tested in the present paper.

Finally we note that, based on optical spectra, no evidence was found in Paper I for a dominant contribution from active galactic nuclei (AGNs) in the sample of 76 distant LIRGs for which a spectroscopic redshift was determined. This confirmed the previous result from Fadda et al. (2001) that AGNs were contributing to less than $20 \%$ of the MIR light of distant LIRGs, as shown by their soft to hard X-ray radiation measured at the Newton and Chandra X-ray observatories.

In this paper, we study the stellar spectra of a sample of 25 LIRGs with a median redshift of $\bar{z}=0.7$ using high-resolution spectroscopy with the FORS2 instrument at the $\operatorname{VLT}(\Delta \lambda / \lambda=1200$, equivalent to 2000 in the rest-frame of the galaxies). This sample is smaller than in Paper I because higher $S / N$ are required to study the continuum emission in comparison to the emission lines. All galaxies are detected at $15 \mu \mathrm{m}$ with ISOCAM and the 11 galaxies located in one of the three fields are also detected at $24 \mu \mathrm{m}$ with the MIPS camera onboard Spitzer (Papovich et al. 2004; Elbaz et al. 2005). We show that both indicators provide consistent estimates of the total IR luminosity of the galaxies, hence also the SFR (see also Elbaz et al. 2005; Marcillac et al. 2005, for more detailed studies).

Section 2 presents the sample selection and the wavelet decomposition technique that we used to analyze the spectra. Section 3 describes the method used in this paper to study the SFR history of the galaxies, namely the Balmer absorption line $\mathrm{H} \delta_{\mathrm{A}}(4101 \AA)$ versus $4000 \AA$ break position of the galaxies, as previously done by Kauffmann et al. (2003) for the Sloan Digital Sky Survey (SDSS). We extended the method used in Kauffmann et al. (2003) to the high-order Balmer lines H8 (3889 $\AA$ ) and H9 (3835 ̊), which are easier to detect in distant galaxies due to the k-correction. The comparison of local and distant LIRGs in this parameter space is discussed in Sect. 4, while the model used to generate Monte Carlo realisations of 200000 spectra with different star formation histories is presented in Sect. 5. The results are presented in Sect. 6 and discussed in the last section.

Throughout this paper, we will assume $H_{0}=$ $75 \mathrm{~km} \mathrm{~s}^{-1} \mathrm{Mpc}^{-1}, \Omega_{\text {matter }}=0.3$ and $\Omega_{\Lambda}=0.7$.

\section{Sample selection and data reduction}

\subsection{Sample selection}

The sample of distant LIRGs was selected from three deep ISOCAM $15 \mu \mathrm{m}$ surveys and is described in Paper I. 105 galaxies were selected on the basis of their $15 \mu \mathrm{m}$ flux density in three different regions of the southern hemisphere, hence avoiding strong contamination by cosmic variance: the ISOCAM UltraDeep Surveys in the FIRBACK (UDSF) and ROSAT (UDSR) fields and the CFRS $3^{\text {h }}$ field. All three fields were selected for their low cirrus contamination and high galactic latitude. The UDSF $\left(9^{\prime} \times 9^{\prime}\right)$ is located at the center of the "far infrared background" survey at $175 \mu \mathrm{m}$ with ISOPHOT onboard ISO ("FIRBACK"; Puget et al. 1999; Lagache \& Dole 2001). The UDSR is centered at the position of a deep ROSAT survey (Zamorani et al. 1999). The UDSR and UDSF are both close to the position of the so-called "Marano Field", originally selected for an optical survey of quasars (Marano et al. 1988), but are separated by $21^{\prime}$ with respect to each other. The third field is one of the Canada France Redshift Survey fields (CFRS $3^{\text {h}}$ ) combining deep infrared, optical, and radio data, as well as spectra from the MOS multiobject spectrograph on the $3.6 \mathrm{~m}$ CFHT.

The ISOCAM sources were selected to span the whole flux density range of the three surveys whose $80 \%$ completeness limits are $150 \mu \mathrm{Jy}$ for the UDSF and UDSR and $250 \mu \mathrm{Jy}$ for the CFRS $3^{\mathrm{h}}$, while the detection limits are $50 \mu \mathrm{Jy}$ (UDSF, UDSR) and $170 \mu \mathrm{Jy}$ (faintest sources in the regime below completeness). No optical selection was applied so that the magnitudes of the sources range from $R_{\mathrm{AB}}=19.4$ to 23.7. At about the same epoch (November 2003) as the VLT-FORS2 observations, a $24 \mu \mathrm{m}$ survey was being performed with the MIPS camera onboard Spitzer during the MIPS commissioning phase (IOC/SV). This survey covers the whole UDSF field and all eleven ISOCAM$15 \mu \mathrm{m}$ sources were detected at $24 \mu \mathrm{m}$, i.e. $45 \%$ of the sample. 
Table 1. Description of the distant LIRGs sample. The technique used to compute the equivalent widths of H $\delta, \mathrm{H} 8, \mathrm{H} 9, \mathrm{H} 10$ and the $4000 \AA$ break (D4000) is described in Sect. 2.3. $L_{\mathrm{IR}}$ and $S F R_{\mathrm{IR}}$ were derived using the Chary \& Elbaz (2001) technique.

\begin{tabular}{|c|c|c|c|c|c|c|c|c|c|c|}
\hline $\begin{array}{l}\text { Object } \\
\text { Slit } \\
\text { (1) }\end{array}$ & $\begin{array}{c}\text { RA } \\
(\mathrm{J} 2000) \\
(2)\end{array}$ & $\begin{array}{c}\text { Dec } \\
(\mathrm{J} 2000) \\
(3)\end{array}$ & (4) & H8 & $\mathrm{H} \delta_{\mathrm{A}}$ & D4000 & $\begin{array}{c}F_{v}^{15 \mu \mathrm{m}} \\
\mu \mathrm{Jy} \\
(8)\end{array}$ & $\begin{array}{c}F_{v}^{24 \mu \mathrm{m}} \\
\mu \mathrm{Jy} \\
(9)\end{array}$ & $\log \left(\frac{L_{\mathrm{IR}}}{L_{\odot}}\right)$ & $\begin{array}{r}S F R_{\mathrm{IR}} \\
M_{\odot} \mathrm{yr}^{-1} \\
(11)\end{array}$ \\
\hline UDSR08 & 31455.20 & -552031.0 & 0.7291 & $3.8 \pm 0.5$ & $4.2 \pm 0.9$ & $1.11 \pm 0.02$ & $236 \pm 75$ & - & $11.44 \pm 0.24$ & 46.7 \\
\hline UDSR09 & 31456.10 & -552008.0 & 0.3884 & $1.4 \pm 0.4$ & - & $1.74 \pm 0.03$ & $609 \pm 87$ & - & $11.20 \pm 0.08$ & 27.2 \\
\hline UDSR 10 & 31443.90 & -552135.0 & 0.6798 & $4.3 \pm 0.6$ & $8.4 \pm 0.7$ & $1.25 \pm 0.03$ & $495 \pm 91$ & - & $11.78 \pm 0.13$ & 102.9 \\
\hline UDSR14 & 31443.30 & -552011.0 & 0.8150 & $4.7 \pm 0.4$ & $3.9 \pm 0.4$ & $1.15 \pm 0.02$ & $200 \pm 69$ & - & $11.48 \pm 0.27$ & 52.0 \\
\hline UDSR 20 & 31441.10 & -551840.0 & 0.7660 & $6.9 \pm 0.5$ & $6.5 \pm 0.8$ & $1.19 \pm 0.02$ & $214 \pm 72$ & - & $11.44 \pm 0.25$ & 47.0 \\
\hline UDSR 23 & 31432.10 & -551902.0 & 0.7094 & $4.9 \pm 0.4$ & $4.7 \pm 0.3$ & $1.15 \pm 0.02$ & $271 \pm 80$ & - & $11.48 \pm 0.22$ & 51.8 \\
\hline UDSF06 & 31330.20 & -550404.0 & 0.6928 & $4.3 \pm 1.3$ & - & $1.27 \pm 0.06$ & $150 \pm 75$ & $180 \pm 22$ & $11.09 \pm 0.49$ & 21.0 \\
\hline UDSF07 & 31317.30 & -550516.0 & 0.7014 & $3.6 \pm 0.9$ & $4.2 \pm 0.7$ & $1.17 \pm 0.04$ & $154 \pm 32$ & $198 \pm 27$ & $11.12 \pm 0.15$ & 22.6 \\
\hline UDSF12 & 31307.70 & -550526.0 & 0.7388 & $7.6 \pm 0.9$ & - & $1.29 \pm 0.06$ & $331 \pm 58$ & $420 \pm 30$ & $11.64 \pm 0.11$ & 74.7 \\
\hline UDSF16 & 31308.0 & -550418.0 & 0.4548 & - & $3.7 \pm 0.6$ & $1.25 \pm 0.01$ & $138 \pm 29$ & $205 \pm 26$ & $10.55 \pm 0.11$ & 6.0 \\
\hline UDSF17 & 31308.60 & -550357.0 & 0.8100 & $6.2 \pm 0.3$ & $6.7 \pm 0.4$ & $1.22 \pm 0.02$ & $257 \pm 47$ & $343 \pm 23$ & $11.62 \pm 0.12$ & 72.0 \\
\hline UDSF18 & 31316.5 & -550227.0 & 0.4620 & - & $4.7 \pm 0.8$ & $1.17 \pm 0.01$ & $170 \pm 44$ & - & $10.68 \pm 0.10$ & 8.2 \\
\hline UDSF19 & 31309.80 & -550308.0 & 0.5476 & $4.8 \pm 1.0$ & $3.9 \pm 1.2$ & $1.32 \pm 0.05$ & $611 \pm 90$ & $778 \pm 40$ & $11.62 \pm 0.09$ & 71.4 \\
\hline UDSF28 & 31251.80 & -550257.0 & 0.6612 & $2.9 \pm 0.6$ & - & $1.47 \pm 0.04$ & $321 \pm 35$ & $861 \pm 37$ & $11.49 \pm 0.06$ & 53.1 \\
\hline UDSF29 & 31250.20 & -550259.0 & 0.6619 & $4.1 \pm 1.3$ & - & $1.37 \pm 0.06$ & $354 \pm 66$ & $568 \pm 28$ & $11.54 \pm 0.11$ & 59.6 \\
\hline UDSF20 & 31319.00 & -550142.0 & 0.8424 & $2.8 \pm 0.7$ & - & $1.33 \pm 0.04$ & $117 \pm 25$ & $248 \pm 21$ & $11.19 \pm 0.16$ & 26.7 \\
\hline UDSF31 & 31244.00 & -550321.0 & 0.6868 & $4.5 \pm 1.1$ & $5.6 \pm 1.1$ & $1.44 \pm 0.06$ & $193 \pm 38$ & $174 \pm 28$ & $11.24 \pm 0.14$ & 29.9 \\
\hline CFRS02 & 30252.01 & +001033.0 & 0.6172 & $6.9 \pm 1.0$ & $5.9 \pm 1.2$ & $1.30 \pm 0.10$ & $582 \pm 114$ & - & $11.75 \pm 0.12$ & 95.8 \\
\hline CFRS06 & 30249.08 & +00101.8 & 0.6169 & $3.8 \pm 0.4$ & $5.0 \pm 0.3$ & $1.21 \pm 0.02$ & $370 \pm 95$ & - & $11.50 \pm 0.17$ & 53.5 \\
\hline CFRS08 & 30246.29 & +001353.6 & 0.7154 & $4.0 \pm 0.3$ & $5.9 \pm 0.9$ & $1.24 \pm 0.03$ & $360 \pm 95$ & - & $11.65 \pm 0.17$ & 75.7 \\
\hline CFRS10 & 30244.57 & +00 1220.1 & 0.5276 & $5.6 \pm 0.6$ & $5.9 \pm 1.2$ & $1.03 \pm 0.04$ & $220 \pm 95$ & - & $11.01 \pm 0.40$ & 17.7 \\
\hline CFRS11 & 30242.31 & +00 129.5 & 0.6895 & - & $2.5 \pm 0.7$ & $1.15 \pm 0.04$ & $425 \pm 110$ & - & $11.70 \pm 0.16$ & 85.4 \\
\hline CFRS14 & 30240.44 & +00143.8 & 0.4652 & $4.6 \pm 0.5$ & $6.5 \pm 0.5$ & $1.10 \pm 0.02$ & $172 \pm 117$ & - & $10.69 \pm 0.58$ & 8.4 \\
\hline CFRS16 & 30238.80 & +00 1417.5 & 0.8274 & $3.3 \pm 1.0$ & - & $1.30 \pm 0.05$ & $444 \pm 100$ & - & $11.99 \pm 0.15$ & 166.7 \\
\hline CFRS29 & 30229.41 & +00 1259.8 & 0.8804 & $7.3 \pm 1.4$ & - & $1.13 \pm 0.05$ & $370 \pm 103$ & - & $11.98 \pm 0.20$ & 163.6 \\
\hline
\end{tabular}

The scan map AOT was used, with an half-array overlap to cover about 1300 sq. arcmin with high redundancy (20) and to get an integration time per sky pixel of about 230s (Papovich et al. 2004). The data were reduced using the Spitzer Science Center Pipeline and the BCD products (Basic Calibrated Data, Pipeline version S10.0.3) were downloaded from the Spitzer archive ${ }^{1}$. The PSF-fitting photometry was performed using DAOPHOT (Stetson 1987) with IRAF ${ }^{2}$.

From a total of 105 galaxies observed with FORS2 at the VLT (Paper I), we detected 3 stars, 13 galaxies were too faint for a redshift determination, and within the remaining list 13 other galaxies were not detected with ISOCAM. The resulting list of 76 galaxies with an MIR detection is divided into 34 "normal" galaxies, 36 LIRGs, and 6 ULIRGs, where "normal" galaxies designates all galaxies except IR luminous galaxies (i.e LIRGs and ULIRGs). The median SFRs associated to each total IR luminosity bin are 4, 54 and $196 M_{\odot} \mathrm{yr}^{-1}$. Because good quality spectra are required to study the stellar absorption lines ( $S / N>3$ on the continuum per resolution element), the final sample that we study in the present paper consists of 25 LIRGs with IR luminosities ranging between $10^{11}$ and $10^{12} L_{\odot}$. The median redshift of the final sample is $\bar{z}=0.7$. The measured properties of the galaxies are summarized in Table 1.

The total $(8-1000 \mu \mathrm{m})$ IR luminosities, $L_{\mathrm{IR}}$, were derived using the library of template SEDs built by Chary \& Elbaz (2001), as in Elbaz et al. (2005). We also computed $L_{\mathrm{IR}}$ from the Dale \& Helou (2001) library following the technique described in Marcillac et al. (2005) and found a median value for $L_{\mathrm{IR}} 10 \%$

\footnotetext{
${ }^{1}$ http://archive.spitzer. caltech.edu, PID: 718

2 IRAF is distributed by the National Optical Astronomy Observatories, which are operated by the Association of Universities for Research in Astronomy, Inc., under cooperative agreement with the National Science Foundation.
}

lower than with the previous library and with an rms of $17 \%$, meanning that both techniques provide consistent luminosities. We then compared the $L_{\mathrm{IR}}$ derived with $15 \mu \mathrm{m}$ and/or $24 \mu \mathrm{m}$ flux densities for the 11 galaxies detected with both ISOCAM and MIPS. Both libraries of template SEDs provide consistent determinations of $L_{\mathrm{IR}}$ using both measurements (with an rms of $30 \%$, see also Elbaz et al. 2005). The median $L_{\mathrm{IR}}$ derived from MIPS is $10 \%$ lower than the one derived from ISOCAM using both libraries, which suggests a possible variation of the MIR spectra of LIRGs as a function of redshift (see Marcillac et al. 2005).

\subsection{Observations and data reduction}

The observations were performed during three nights with FORS2 on the ESO-VLT with the combination of the grisms R600 and I600 ( $3 \mathrm{~h}$ per grism) to cover the wavelength range 5000 to $9200 \AA$ at a resolution of $5 \AA(R=1200)$. At the median redshift of the objects of $\bar{z} \sim 0.7$, the resolution is equivalent to $3 \AA(R=2000)$.

Because absorption lines are wider than emission lines, it is possible to increase the $S / N$ ratio on the absorption lines by working at a lower frequency than the raw spectrum. The physical origin of the width of the absorption lines results from the complex combination of the internal dynamics of the stars and the global dynamics of the galaxy. In order to optimize the line extraction, we therefore decomposed the raw spectra into eight different wavelet scales (Table 2) using the undecimated (keeping an identical sampling in each wavelet scale) wavelet transform (à trous algorithm, Starck \& Murtagh 1994; Starck et al. 2002). We optimized the $S / N$ ratio on the absorption line features by selecting the best combination of wavelet scales. In the wavelet space, the first scale (highest frequency), which we index on scale 0 , corresponds to features of size $0.7 \AA$, while 
Table 2. Definition of the wavelet scales used in the wavelet decomposition of the VLT-FORS2 spectra.

\begin{tabular}{cc}
\hline \hline Wavelet scale & Resolution $(\AA)$ \\
\hline 0 & 0.7 \\
1 & 1.4 \\
2 & 2.8 \\
3 & 5.6 \\
4 & 11.2 \\
5 & 22.4 \\
6 & 44.8 \\
7 & 89.6 \\
\hline
\end{tabular}

features at scale $j$ have a size of $0.7 \times 2^{j} \AA$. Note that the lowest frequency scale is equivalent to the raw spectrum smoothed by a B-spline (equivalent to a Gaussian) of width $89.5 \AA$, i.e. the baseline, while at higher frequencies, each wavelet scale " $i$ " is equal to the difference between the raw spectrum smoothed at the scales " $i$ " and " $i-1$ ". Hence the sum of all wavelet scales plus the baseline (here at scale 7) is exactly equal to the initial raw spectrum.

In order to determine the number of scales to take into account in the decomposition, we started from the lowest resolution in the wavelet space, equivalent to a spectral resolution of $89.5 \AA$. We then kept doubling the frequency level as long as the $S / N$ ratio was increased. This happened on the wavelet scale " 4 " (equivalent to $11.2 \AA$ ), so we used the co-addition of the 4 , 5,6 , and 7 wavelet scales (from 11.2 to $89.6 \AA$, increasing the frequency by a factor 2 for each scale) in order to reconstruct spectra devoid of high-resolution noise. The steps of the decomposition are shown in Fig. 1 for a LIRG, UDSR23 $(z=0.7094)$, located at the median redshift of the sample. The spectra resulting from the combination of these four scales present the advantage of seeing the same spectral resolution as the one used by Bica \& Alloin (1986). The final result is compared to the raw spectrum in the Fig. 2.

Note that the wavelet decomposition can dilute emission lines and that we have checked that absorption lines were not affected by a similar effect. Being wider, they are naturally less affected by this technique. However, we quantified this effect using some stellar spectra extracted from STELIB (Le Borgne et al. 2003) which resolution is about the same as the rest-frame one for the distant LIRGs. We added a white noise to the stellar spectra to reach a $S / N$ ratio of $S / N \sim 3$ on the continuum, interpolated the spectra to reach a resolution of $0.7 \AA$, and applied the same wavelet decomposition to them as for the distant LIRGs. The equivalent widths of the Balmer absorption lines determined before and after the wavelet analysis differ by less than $4 \%$. We included this weak difference in the equivalentwidth uncertainties.

\subsection{Measurement of the Balmer absorption line indices and $4000 \AA$ break}

In the following, we compare the equivalent widths measured for $\mathrm{H} \delta(4101 \AA)$ and for the high-order Balmer absorption line H8 (3889 ̊). We considered using the H9 (3835 $\AA$ ) line that we therefore also define in this section, but it appeared to be a less reliable tracer of the past star formation activity of galaxies, as we discuss in the next section.

We used the definition of the $\mathrm{H} \delta$ pseudo-equivalent width indice as defined in the Lick system (Worthey \& Ottaviani 1997; see Table 3). Since no Lick indices have been defined yet for the high order Balmer absorption lines, we adapted the windows defined by Bica \& Alloin (1986) to the Lick index method for H8 and H9: for each line, the index continuum, the blue, and red bandpasses for each pseudo-continuum are summarized in Table 3. The two lines present the advantage of being located at a lower wavelength to be accessible to higher redshifts in the observed optical range and to be less affected by the overlying nebular emission lines at the same wavelengths.

The $\mathrm{H} \delta$ absorption line is surrounded by iron absorption lines that affect both its red and blue pseudo continua and are responsible for the negative EW measured for this line after a few Gyears, as discussed in the next section. In order to avoid such pollution from neighboring lines, the red continuum of $\mathrm{H} 8$ was chosen to minimize the sensitivity to the CaII H (3933 $\AA$ ) and K (3966 ̊) lines, while the 3855-3865 $\AA$ region is not known to be affected by metallic lines. As a result this index is weakly affected by metallicity. Because no metallic line is located near it, H9 is the least polluted line but is somewhat fainter than $\mathrm{H} 8$ and is surrounded by the strong absorption of $\mathrm{H} 8$ and $\mathrm{H} 10$, which makes the two pseudo continua more difficult to define. We have primarily used $\mathrm{H} \delta_{\mathrm{A}}$ and $\mathrm{H} 8$, which are better determined for most of our spectra but also checked that we obtained consistent results based on the $\mathrm{H} 9$ and $\mathrm{H} \delta$ lines when they were available. We will discuss the results obtained with the Balmer absorption lines $\mathrm{H} \delta_{\mathrm{A}}(4102 \AA)$ and $\mathrm{H} 8(3889 \AA)$ separately. The advantage of this choice is that it provides two independent estimates of the parameters that we are deriving and can be used as a test of the robustness of the Bruzual \& Charlot (2003) code that we are using.

There is an ongoing debate about possible misinterpretation of the equivalent width of the $\mathrm{H} \delta_{\mathrm{A}}$ line because of metallicity ratios that could affect its neighboring regions, hence its associated pseudo-continua (see Thomas et al. 2004; Korn et al. 2005). On the one hand, $\mathrm{H} \delta_{\mathrm{A}}$ has the advantage of allowing the comparison with studies of local galaxies such as SDSS galaxies (Kauffmann et al. 2003), while H8 is usually not available for local galaxies because it lies in a bluer region of the spectrum. On the other hand, the H8 line is not known to be affected by neighboring metallic lines and, as it is located in a bluer region of the spectrum, it is easier to measure for distant galaxies (less polluted by sky emission lines).

Before measuring these absorption features, we corrected them from the overlying nebular emission line whenever possible, as indicated in the paper. The nebular emission lines are not detected directly from the spectra, because they are too faint, but we computed their emission based on the observed $\mathrm{H}_{\beta}$ and $\mathrm{H}_{\gamma}$ emission lines (and $\mathrm{H}_{\alpha}$ for the low- $z$ galaxies) assuming a line ratio corresponding to a case $\mathrm{B}$ recombination for electron densities $\leq 10^{4} \mathrm{~cm}^{-3}$ and temperatures $T_{\mathrm{e}} \sim 10^{4}$ (Osterbrock 1989). The Balmer emission line ratio was also used to compute the attenuation of these lines before subtracting them from the absorption lines measurements. The computation and values for these attenuations can be found in Paper I.

Following the same strategy as Kauffmann et al. (2003), we used the method explained in Bruzual (1983) and the blue and red bandpass definitions introduced by Balogh et al. (1999). The latter are narrower than the ones originally defined by Bruzual (1983) and present the advantage of being less sensitive to reddening effects.

The H8 and D4000 values for each distant LIRG are summarized in Table 1. The scientific interpretation is discussed in the following section. 

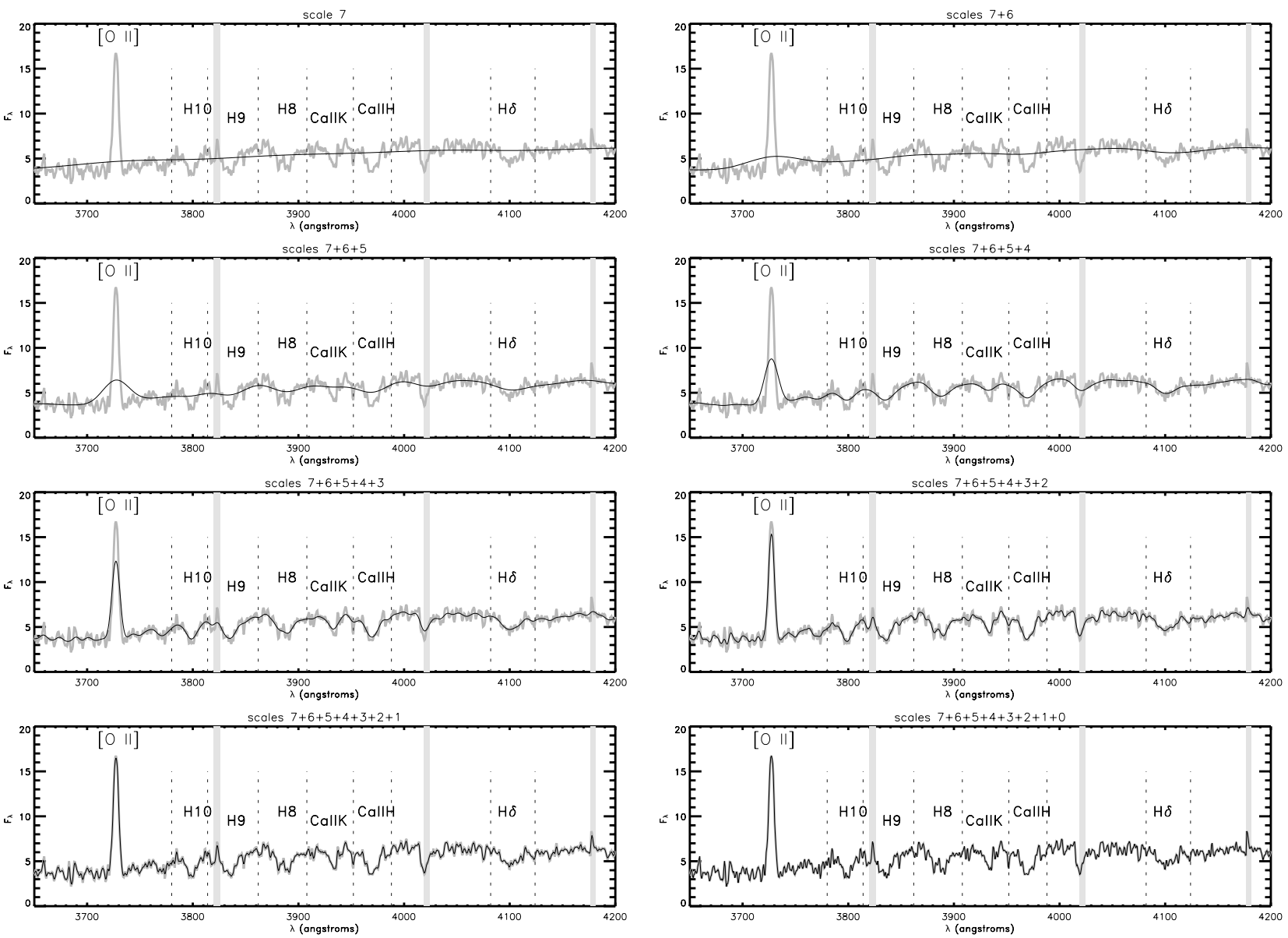

Fig. 1. Wavelet decomposition of the VLT-FORS2 spectrum of a distant LIRG (UDSR23) located at a redshift of $z=0.7094$ ) and forming stars at a rate of $S F R_{\mathrm{IR}}=51.8 M_{\odot} \mathrm{yr}^{-1}$. The raw spectrum (thick grey line) is compared to its wavelet decomposition (thin dark line), starting from scale 7, corresponding to the spectrum smoothed with a B-spline (equivalent to a gaussian) at the lowest resolution of $89.6 \AA$, then adding up the wavelet scales by increasing the spectral resolution from $44.8 \AA$ (scale 6) to $0.7 \AA$ (scale 1), by dividing it by a factor 2 for each scale. The narrow vertical grey zones were masked in the analysis because of the presence of sky emission lines. The vertical dotted lines indicate the minimum and maximum wavelength for each absorption line. In this zoom, the absorption lines clearly appear as much shallower than the emission line in OII.

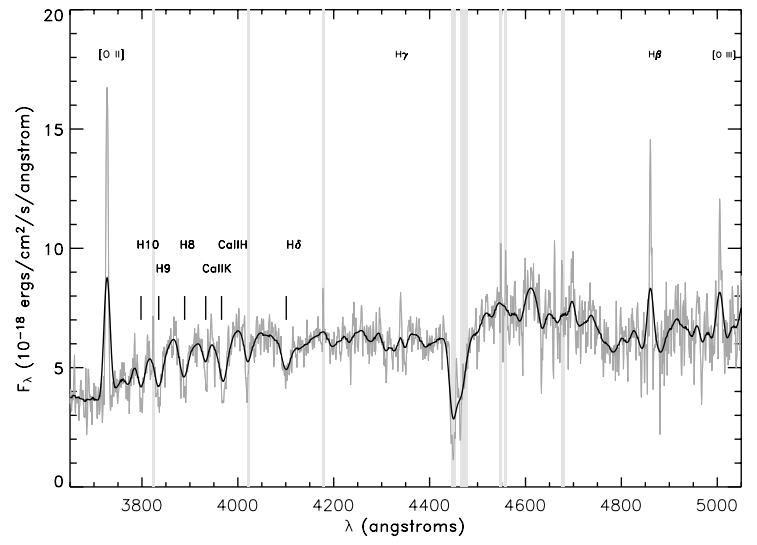

Fig. 2. Comparison of the VLT-FORS2 raw spectrum (grey line) of the distant LIRG UDSR23 $\left(z=0.7094,51.8 \mathrm{M}_{\odot} \mathrm{yr}^{-1}\right)$ to its wavelet decomposition resulting from the combination of the four wavelet scales 4-7, i.e. from 11.2 to $89.6 \AA$ (black line). While the absorption lines are clearly distinguishable, the emission lines such as [OIII], [OII], $\mathrm{H} \beta, \mathrm{H} \gamma$ are partly diluted by this technique.

\section{3. $4000 \AA$ A break and Balmer absorption lines as tracers of the recent star formation history}

In this section, we describe how Balmer absorption lines and the $4000 \AA$ break trace the recent star formation history of galaxies.
For this purpose, we have synthesized a single stellar population (SSP) using the latest version of the "GALAXEV" code from Bruzual \& Charlot (2003). For the moment, we do not include dust attenuation so as to simplify the discussion. Note, however, that the wavelength range over which the equivalent widths and the $4000 \AA$ break are measured is small which implies a marginal correction due to dust attenuation. Nonetheless, we will include dust attenuation in the Monte Carlo realizations that we will compare to the LIRGs and discuss its effects on our findings.

This version of the GALAXEV code includes the spectral library STELIB (Le Borgne et al. 2003) whose spectral resolution is $3 \AA$ from 3200 to $9500 \AA$, which is comparable to the present spectra in the rest-frame of the galaxies. Figure 3 presents the evolution with time of the high order Balmer absorption lines $\mathrm{H} 8$ and $\mathrm{H} 9$, as well as the $\mathrm{H} \delta_{\mathrm{A}}$ absorption line and the $4000 \AA$ break. The four lines in each plot correspond to four different metallicities with the following metal mass fractions (total mass in elements heavier than hydrogen and helium over the mass in hydrogen): $Z=0.004$ (20\% solar, dashed line), $Z=0.008(40 \%$ solar, dash-dotted line), $Z=0.02$ (solar, solid line), $Z=0.05$ (2.5 times solar, dotted line).

The D4000 is the sudden onset of stellar photospheric opacity shortward of $4000 \AA$. It reflects the mean temperature of the stars responsible for the continuum: the metals located in the 
Table 3. Definition of the pseudo-equivalent width indices for the $\mathrm{H} \delta$ $\left(\mathrm{H} \delta_{\mathrm{A}}\right.$ in Worthey \& Ottavianni 1997) and for the two high order Balmer absorption lines. For the two last lines, we followed the same principle as for Lick indices while using windows defined Bica \& Alloin (1986).

\begin{tabular}{ccc}
\hline \hline Name & Blue and red bandpasses $(\AA)$ & Index continua $(\AA)$ \\
\hline $\mathrm{H} \delta_{\mathrm{A}}$ & $4041.60-4079.754128 .50-4161.00$ & $4083.50-4122.25$ \\
$\mathrm{H} 9$ & $3810-38203855-3865$ & $3825-3845$ \\
$\mathrm{H} 8$ & $3855-38653905-3915$ & $3870-3900$ \\
\hline
\end{tabular}

atmosphere of $\mathrm{O}$ and $\mathrm{B}$ stars are more ionized and produce a weaker opacity, hence a smaller $4000 \AA$ break, than those in cooler stars (Bruzual 1983; Poggianti \& Barbaro 1997; Gorgas et al. 1999; Kauffmann et al. 2003). As a result D4000 keeps increasing as a function of the aging of the stellar population (see Fig. 3d). D4000 is sensitive to metallicity as is shown in Table 3d where it varies by more than $20 \%$ after a few billion years or when it is larger than 1.6. As see in the next section, the distant LIRGs have D4000 $=1.2 \pm 0.07$, and therefore metallicity effects are negligible for them. Even if the slope of D4000 versus age is less flat than for young population, i.e. 7 Gyears, it is flat enough to provide uncertain stellar ages if used alone.

In order to trace back the recent star formation history of galaxies, it is therefore necessary to use another tracer of stellar age such as the Balmer absorption lines, which exhibit a steep slope as a function of stellar ages in this range of ages (see Figs. $3 \mathrm{a}-\mathrm{c}$ ). Balmer absorption lines are mainly produced by the atmosphere of A to F stars. However, O and B stars, which do not exhibit strong absorption lines, indirectly affect them by increasing the continuum level and therefore diluting them, which explains the flat values for the equivalent widths of $\mathrm{H} 8, \mathrm{H} 9$, and $\mathrm{H} \delta_{\mathrm{A}}$ in the first few million years (lifetime of $\mathrm{O}$ and $\mathrm{B}$ stars). The rapid increase that follows is produced by the dominant role of $\mathrm{A}$ and $\mathrm{F}$ stars, which then disappears after $\sim 0.5$ Gyear producing the rapid decline of the equivalent widths in Figs. 3a-c. Here again, it is worthwhile noticing the marginal role played by the metallicity in the evolution of the Balmer lines EW with time. The EW varies by less than $20 \%$ a few billion years after the burst as a function of metallicity.

\section{Comparison of local and distant LIRGs}

A sample of 401 local $(z \leq 0.25)$ LIRGs detected with IRAS with optical spectra from the SDSS and emission lines typical of star forming galaxies, as opposed to AGNs, was identifed by Pasquali et al. (2005). The locus of the D4000 and EW $\left(\mathrm{H} \delta_{\mathrm{A}}\right)$ measured for the distant LIRGs is compared to that of local LIRGs and of the field SDSS galaxies in Fig. 4. First, note the concentration of both LIRG populations at low D4000 and high $\mathrm{H} \delta_{\mathrm{A}}$, which suggests that local and distant LIRGs share a similar recent star formation history. We could not produce similar figures for high-order Balmer absorption lines for this comparison since they are too blue to be accessible in the SDSS spectra. Their position in the $\mathrm{D} 4000-\mathrm{H} \delta_{\mathrm{A}}$ diagram indicates that the young stellar population that is producing the large IR luminosity is not completely obscured by dust in the optical because the median D4000 value of 1.2 for these galaxies correspond to stellar ages lower than 1 Gyear, which is much below the ages of these galaxies (see Table 4). This already suggests that even in these dusty galaxies the optical spectral signatures can be used as a tracer of the recent star formation history. The quasi absence of distant LIRGs above D4000 $=1.25$, where half of the local LIRGs lie, suggests that the distant LIRGs are younger than the local ones.
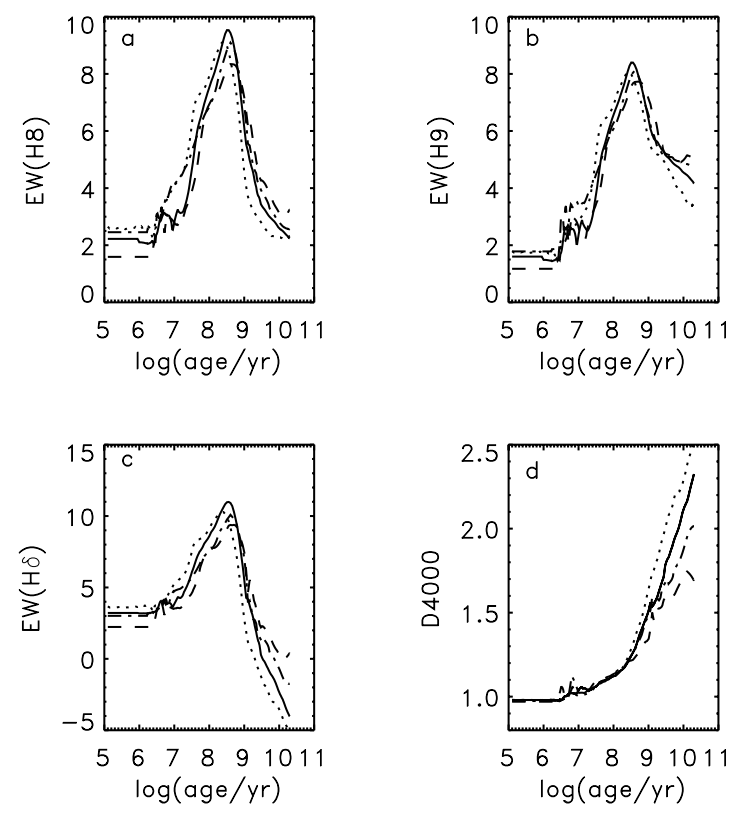

Fig. 3. Time evolution of the $\mathrm{H} 8, \mathrm{H} 9$, and $\mathrm{H} \delta_{\mathrm{A}}$ indices and $4000 \AA$ break (D4000) for a single stellar population synthesized with the GALAXEV code (Bruzual \& Charlot 2003). Four different metallicities were used in each plot, demonstrating the marginal effect of metallicity on the three indices and on D4000 before 1 Gyear: $Z=0.004$ (20\% solar, dashed line), $Z=0.008$ (40\% solar, dash-dotted line), $Z=0.02$ (solar, solid line), $Z=0.05$ (2.5 times solar, dotted line). From top to bottom and left to right: a) $\mathrm{H} 8$ evolution; b) $\mathrm{H} 9$ evolution; c) $\mathrm{H} \delta_{\mathrm{A}}$ evolution; d) D4000 evolution.

The relative proportion of young and old stellar populations can be studied in these galaxies by comparing the stellar masses, derived mostly from the old stellar population dominating in the near infrared range, with their D4000 values. Massive galaxies are generally older as shown by Fig. 5, where the stellar mass of SDSS galaxies increases with D4000. The position of the local LIRGs in Fig. 5 suggests that they are massive galaxies that were located on the right side of the plot before the burst and were shifted to the left during the burst, which decreased their D4000 value. This figure reinforces our interpretation that the low value of D4000 for the LIRGs is due to the addition of a young population on top of an older population. The comparison of field SDSS galaxies with IRAS LIRGs in four bins of D4000 from 1 to 1.4 (bin size $0.1,68 \%$ error bars) shows that local LIRGs exhibit systematically lower D4000 values for their stellar masses.

The incompleteness of the photometric data for our sample of galaxies prevented the determination of their stellar masses. However, another sample of mid IR selected LIRGs at $\bar{z}=$ 0.7 located in the Hubble Deep Field South was studied by Franceschini et al. (2003), who computed their stellar masses using a Salpeter IMF and a combination of single stellar populations with ten different ages, to fit their UV-optical-NIR spectra of IR luminous galaxies. From their Table 6, a total of 14 LIRGs possess a spectroscopic redshift between $z=0.4$ and 1.2, and 7 more have a photometric redshift in this range. After converting Franceschini's values to $H_{0}=75 \mathrm{~km} \mathrm{~s}^{-1} \mathrm{Mpc}^{-1}$, we find a median stellar mass of $7 \times 10^{10} M_{\odot}$ for the 14 galaxies with a spectroscopic redshift. Including the less robust photometric redshifts only changes this value to $6 \times 10^{10} M_{\odot}$. Combined with a median D4000 1.2, this places the distant LIRGs in a very similar locus as the local LIRGs (see Fig. 5). Their low D4000 values are therefore also representative of the young stellar 


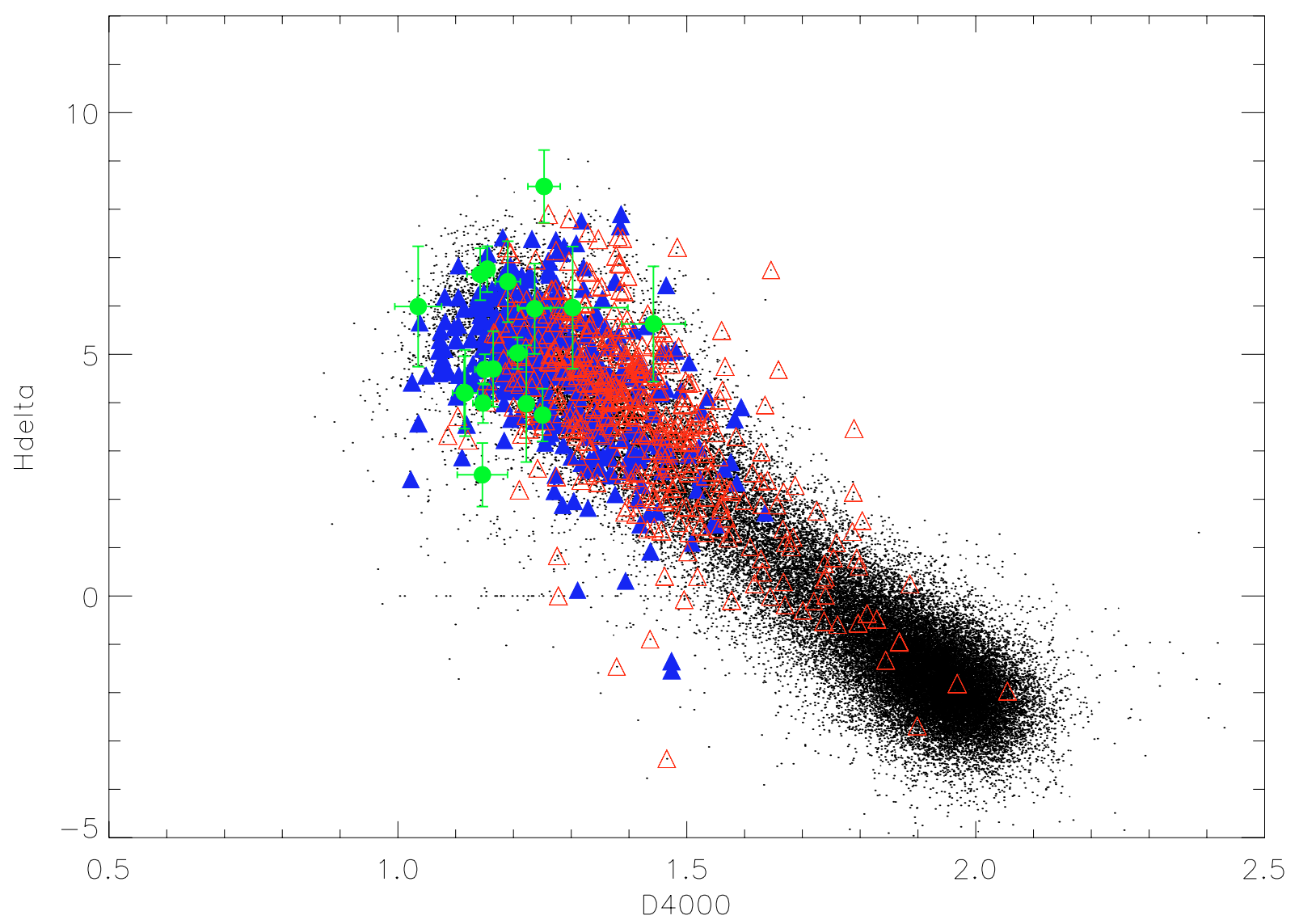

Fig. 4. $\mathrm{H} \delta_{\mathrm{A}}$ versus D4000 diagram for the SDSS galaxies (black points) and distant LIRGs of the present sample (green filled circles with error bars). The sub-population of SDSS galaxies detected with IRAS are marked with triangles and separated into galaxies with optical spectra with (empty red triangles) and without (filled blue triangles) an AGN signature.

Table 4. Median and $68 \%$ dispersion (around the median) of the D4000 and $\mathrm{H} \delta_{\mathrm{A}}$ indices for local and distant galaxies.

\begin{tabular}{ccc}
\hline \hline Indice & Distant LIRGs & Local LIRGs \\
\hline $\mathrm{D} 4000$ & $1.2_{-0.07}^{+0.07}$ & $1.25_{-0.08}^{+0.12}$ \\
$\mathrm{H} \delta_{\mathrm{A}}$ & $5.0_{-1.1}^{+1.5}$ & $4.9_{-1.5}^{+1.07}$ \\
\hline
\end{tabular}

population of the burst superimposed on top of an older stellar population dominating the stellar mass of the galaxies.

\section{Description of the model}

We used the Bruzual \& Charlot (2003) model to synthesize a series of 200000 Monte Carlo realizations including various star formation histories. Although the technique is similar to the one used in Kauffmann et al. (2003), it presents two differences related to the populations of galaxies that we are studying here, i.e. dusty starbursts. The priors of the model were set to include a range of dust attenuations and to include a larger fraction of starbursting galaxies. We will discuss the effects of both modifications in the following.

Each star formation history was modeled with eight parameters:

- the age of the galaxy since its formation, $t_{\text {form }}$. We imposed a maximum age of 7 Gyear, which is the age of the universe at the median redshift of the galaxies, $\bar{z} \sim 0.7$;

- the parameter $\gamma$ for the underlying "continuous" SFR (as opposed to the starburst itself): $S F R^{\text {cont }}(t) \propto \mathrm{e}^{-\gamma t}$;
- the $S F R$ of the burst itself, $S F R^{\text {burst }}$, is not a parameter, but it results from the combination of two parameters: the burst duration, $\tau_{\mathrm{B}}$, and the stellar mass fraction produced during the burst, $f_{\mathrm{B}}$. Note that $f_{\mathrm{B}}$ computed in the model is the ratio of the stellar mass formed during the burst, $M_{\star}^{\text {burst }}$, over the total stellar mass formed through continuous star formation during $t_{\text {form }}, M_{\star}^{\text {cont }}$. Here $M_{\star}^{\text {cont }}$ is not corrected for the mass returned to the interstellar medium by evolved stars and is therefore larger than the actual stellar mass of the galaxy, $M_{\star}$. In the following, we call, $f_{\mathrm{B}}^{\text {eff }}$, the "effective burst stellar mass fraction", computed with $M_{\star}$ instead of $M_{\star}^{\text {cont; }}$

- the dust attenuation parameters, $\mu$ and $\tau_{\mathrm{V}}$, defined as in Charlot \& Fall (2000), i.e. a differential attenuation function of the age of the stars (see Eq. (1) and Fig. 6a). Stars younger than $10^{7}$ years, the typical lifetime of a giant molecular cloud (GMC), are assumed to be embedded in their dusty parent GMC with an optical depth $\tau_{\mathrm{V}}$. Older stars are supposed to have escaped their parent GMC and their ambient medium's optical depth is assumed to be $\mu$ times smaller. This attenuation law was chosen because it reproduces well the correlation observed for UV selected starbursts of the FIR over UV ratio with the UV slope, $\beta$, as well as the $L_{\mathrm{H} \alpha}$ over $L_{\mathrm{H} \beta}$ ratio. The optical depth for stars younger than 0.1 Gyears, $\tau_{\mathrm{V}}$, was set between 1 and 4 based on the observed values for LIRGs (Liang et al. 2004; Flores et al. 2004), while $\mu$ was allowed to vary from 0.1 to 1 :

$$
\begin{array}{ll}
F_{\lambda}^{\text {observed }}=F_{\lambda}^{\text {intrinsic }} \times \mathrm{e}^{-\tau_{\lambda}} & \\
\tau_{\lambda}=\tau_{\mathrm{V}} \times(5500 / \lambda)^{0.7} & t_{\star}<10^{7} \mathrm{yr} \\
\tau_{\lambda}=\mu \times \tau_{\mathrm{V}} \times(5500 / \lambda)^{0.7} & t_{\star} \geq 10^{7} \mathrm{yr}
\end{array}
$$




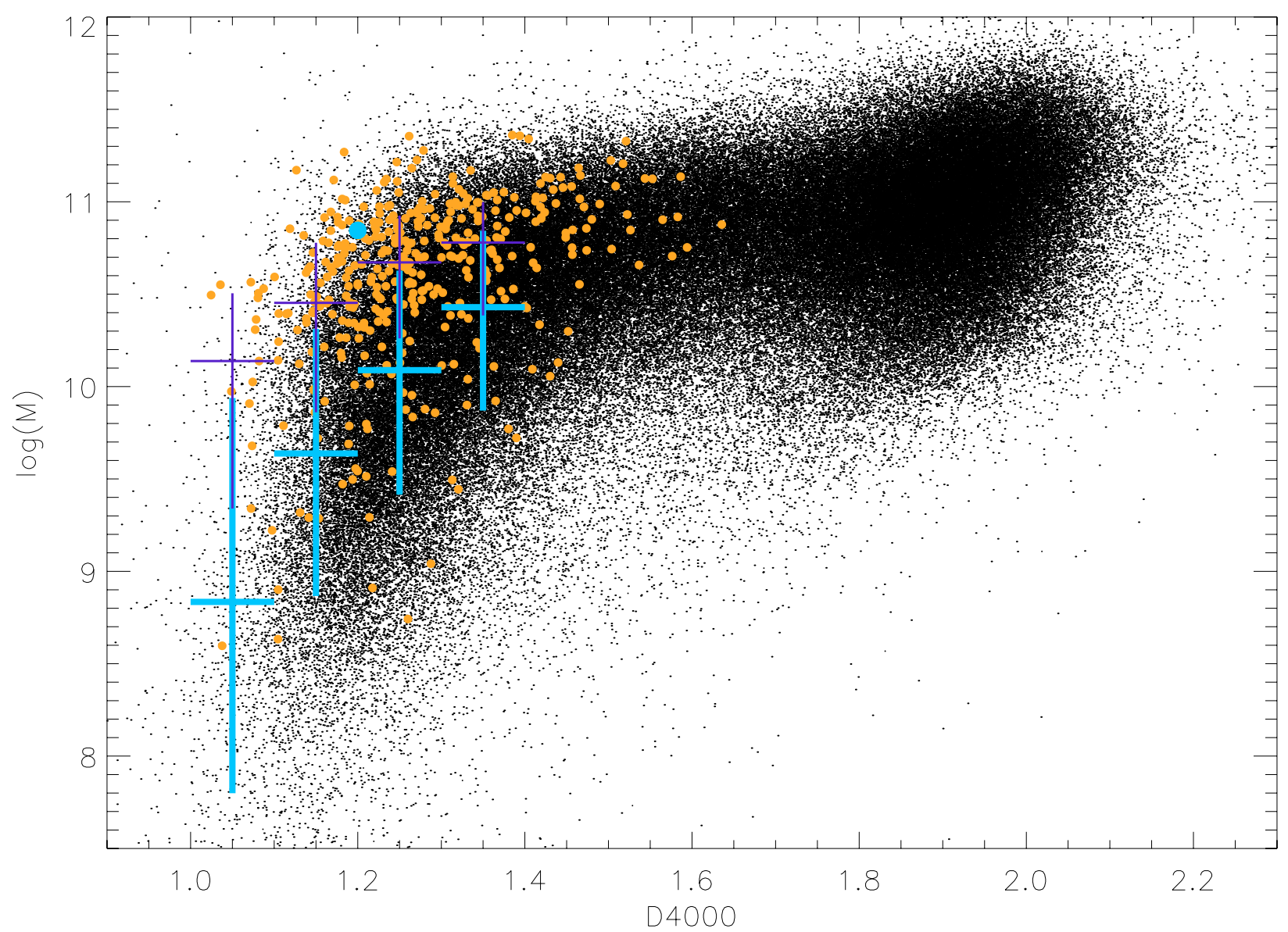

Fig. 5. Stellar mass as a function of D4000 for the SDSS galaxies (black points) and distant LIRGs of the present sample (large filled blue circle). The median stellar mass for the distant LIRGs was derived from Franceschini et al. (2003) as discussed in the text. The sub-population of SDSS galaxies detected with IRAS and without an AGN signature in their optical spectra (star-forming galaxies) are marked with orange filled circles. The median and 1- $\sigma$ error bars for the field SDSS galaxies are represented with thick light-blue crosses, while the thin dark-blue crosses are for the sub-sample of star-forming SDSS-IRAS galaxies.

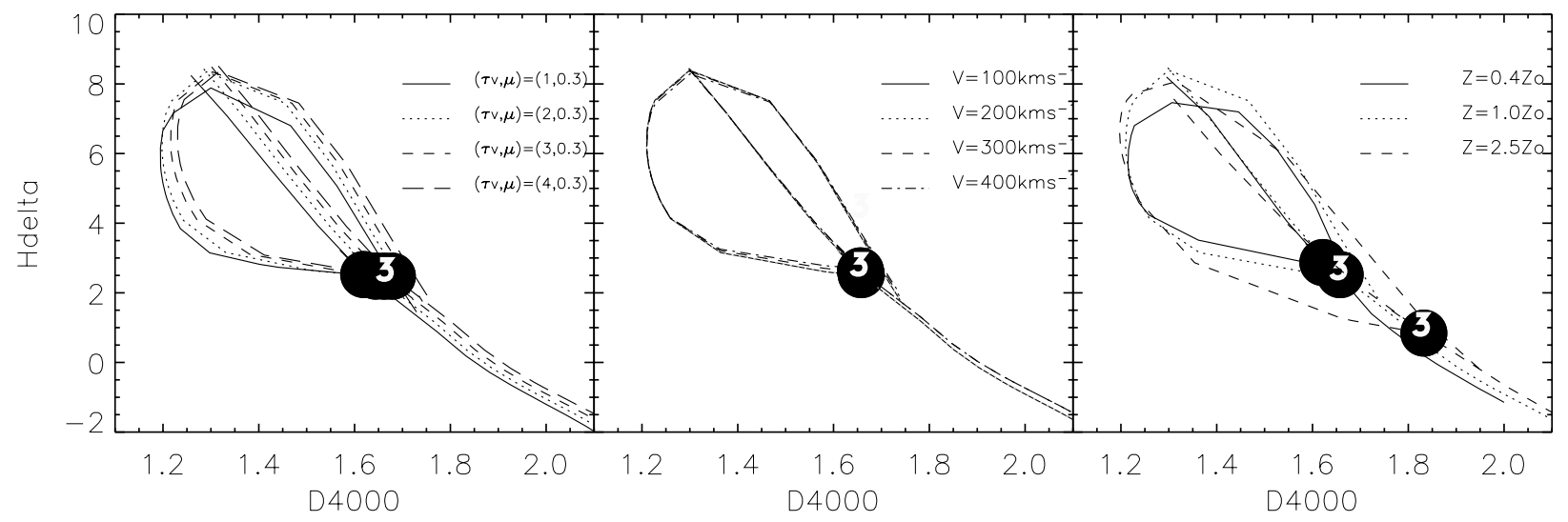

Fig. 6. Influence of the attenuation, velocity dispersion, and metallicity for a typical star formation history synthesized with the GALAXEV code (Bruzual \& Charlot 2003). In all three plots, the loops correspond to a burst of star formation that started 3 Gyear (big black circles) after the

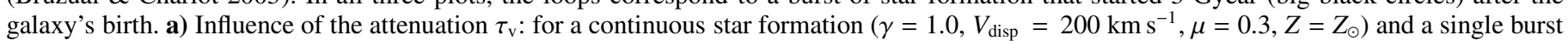
$\left(\tau_{\mathrm{B}}=0.1\right.$ Gyear and $\left.f b=10 \%\right)$; the solid line: $\tau_{\mathrm{v}}=1.0$. The dotted line: $\tau_{\mathrm{v}}=2.0$. The dashed line: $\tau_{\mathrm{v}}=3.0$. The long dashed line: $\left.\tau_{\mathrm{v}}=4.0 . \mathbf{b}\right)$ Influence of the velocity dispersion $V_{\text {disp }}$ : for a continuous star formation $\left(\gamma=1.0, \tau_{\mathrm{v}}=3.0, \mu=0.3, Z=Z_{\odot}\right)$ and a single burst $\left(\tau_{\mathrm{B}}=0.1 \mathrm{Gyear}\right.$ and $f b=10 \%)$. The solid line: $V_{\text {disp }}=100 \mathrm{~km} \mathrm{~s}^{-1}$. The dotted line: $V_{\text {disp }}=200 \mathrm{~km} \mathrm{~s}^{-1}$. The dashed line: $V_{\text {disp }}=300 \mathrm{~km} \mathrm{~s}$. c) Influence of the metallicity $Z$ : for a continuous star formation $\left(\gamma=1.0, V_{\text {disp }}=200 \mathrm{~km} \mathrm{~s}^{-1}, \tau_{\mathrm{v}}=3.0\right.$ and $\left.\mu=0.3\right)$ and a single burst $\left(\tau_{\mathrm{B}}=0.1 \mathrm{Gyear}\right.$ and $\left.f b=10 \%\right)$. The solid line: $Z=0.5 Z_{\odot}$. The dotted line: $Z=Z_{\odot}$. The dashed line: $Z=2.5 Z_{\odot}$.

- the velocity dispersion, $V_{\text {disp. }}$. We allowed a wide range of velocity dispersions to test the effect of a broadening of the $\mathrm{H} \delta_{\mathrm{A}}$ absorption lines, which would mimick the intrinsically large width of the absorption lines due to the combination of the internal dynamics of the stars with the mass of the galaxy. The influence of the velocity dispersion on the position of a 
Table 5. Description of the range of values used as priors for the simulations.

\begin{tabular}{cc}
\hline \hline \multicolumn{3}{c}{ Continuous star formation } \\
$t_{\text {form }}\left(\right.$ Gyear $\left.^{2}\right)$ & $1-7$ \\
$\gamma\left(\right.$ Gyear $\left.^{-1}\right)$ & $0-3$ \\
\hline \multicolumn{2}{c}{ Metallicity \& velocity dispersion } \\
$Z / Z_{\odot}$ & $0.5-1.5$ \\
$V_{\text {disp }}\left(\mathrm{km} . \mathrm{s}^{-1}\right)$ & $100-300$ \\
\hline \multicolumn{2}{c}{ Attenuation } \\
$\tau_{\mathrm{V}}$ & $1-4$ \\
$\mu$ & $0.1-1.0$ \\
\hline Burst fraction and duration \\
$f_{\mathrm{B}}$ \\
$\tau_{\mathrm{B}}\left(10^{8} \mathrm{yr}\right)$ & $0-1$ \\
\multicolumn{2}{c}{}
\end{tabular}

galaxy in the $\mathrm{H} \delta_{\mathrm{A}}-\mathrm{D} 4000$ diagram was found to be negligible (see Fig. 6b);

- the metallicity, $Z$. The influence of the stellar metallicity is stronger than that of the velocity dispersion but remains smaller than the error bars on the measured $\mathrm{H} \delta$ and $D 4000$ (see Fig. 6c).

Table 5 summarizes the range over which those eight parameters were chosen, the so-called priors, which represent the key adjustment when using Bayesian statistics. The goal of these simulations is not to constrain all eight parameters, and indeed some of them produce competitive effects or cannot be disentangled:

- a galaxy with a given age and $\gamma$ parameter for its $S F R$ will be located at the same position in the H8-D4000 diagram than a younger galaxy with a higher value for $\gamma$;

- the two parameters used in the attenuation law, $\mu$ and $\tau_{\mathrm{V}}$. Figure 6 shows that galaxies are shifted towards the upperright of the $\mathrm{H} \delta_{\mathrm{A}}-\mathrm{D} 4000$ (or equivalently H8-D4000) diagram with increasing dust attenuation. This is an important point when comparing dusty galaxies to field galaxies: even though LIRGs overlap with a fraction of SDSS field galaxies in Fig. 4, their recent star formation history is interpreted differently if their dust attenuation is taken into account.

Instead, we will use a Bayesian approach such as the one detailed of Kauffmann et al. (2003) to derive the following characteristics of the starbursts:

1. the Scalo parameter, i.e. the ratio of present to past-averaged star formation rate. This parameter defines the relative intensity of the present starburst;

2. the burst duration, $\tau_{\mathrm{B}}$;

3. the last burst stellar mass fraction, $f_{\mathrm{B}}$ (and $f_{\mathrm{B}}^{\mathrm{eff}}$ ).

The Bayesian statistics approach consists of determining a probability distribution function (PDF) for any one of these parameters, which consists of a histogram of the number of Monte Carlo realizations weighted by the probability function $\exp \left(-\chi^{2} / 2\right)$, where $\chi^{2}$ is defined in Eq. (2). The convergence of the technique is reached if the PDF is peaked, and its shape provides the precision of the determination:

$\chi^{2}=\left(\frac{\mathrm{H} \delta^{\mathrm{obs}}-\mathrm{H} \delta^{\mathrm{sim}}}{\sigma\left(\mathrm{H} \delta^{\mathrm{obs}}\right)}\right)^{2}+\left(\frac{\mathrm{D} 4000^{\mathrm{obs}}-\mathrm{D} 4000^{\mathrm{sim}}}{\sigma\left(\mathrm{D} 4000^{\mathrm{obs}}\right)}\right)^{2}$.

\section{Results}

\subsection{Signature of the presence of a starburst in the distant LIRGs in the H8-D4000 diagram}

We generated three series of simulations with the same priors but varying the percentage of galaxies experiencing a starburst at the age of the simulated spectrum. In a first simulation, SIM1, only $20 \%$ of the Monte Carlo realizations include ongoing starburst. The positions in the H8-D4000 diagram of the 200000 Monte Carlo realizations of SIM1 are illustrated in Fig. 7. The light blue points on the lower-left part of the figure illustrate the position of galaxies with an ongoing burst of star formation. The upper dark blue points correspond to post-starbursts, i.e. galaxies having experienced a recent starburst which ended less than 2 Gyear ago, and dark points to galaxies with continuous star formation or at least no burst during the past 2 Gyears. The lines illustrate the tracks followed by an individual galaxy during its lifetime: a galaxy with purely continuous star formation will follow the bold red line, where the numbers mark its age in Gyear $\left(\gamma=2, \mu=0.3, \tau_{\mathrm{V}}=3.0, V_{\text {disp }}=200 \mathrm{~km} \mathrm{~s}^{-1}\right)$. If this galaxy was experiencing a burst of star formation when it was 3 Gyears old, then it would follow one of the loops in light orange towards the left of the diagram for a total duration of 2 Gyears and then go back to the track of a galaxy with pure continuous star formation, having lost the memory of its past starburst. During the loop, D4000 is at first the most affected by the presence of young stars, moving towards the left of the diagram; then it is $\mathrm{H} 8$ which increases moving upwards in the diagram, before the galaxy slowly goes back to the continuous regime. The first part of the loop indicates that the galaxy is still bursting, while the remaining part of the loop corresponds to the post-starburst regime. We have plotted a loop starting at an age of 2 Gyears (light orange) and another starting at 3 Gyears (dark orange). In each case we computed the track of $\tau_{\mathrm{B}}=5 \times 10^{7}$ years (dashed lines) and $10^{8}$ years (plain line) starburst with a fraction of $f_{\mathrm{B}}=15 \%$.

$75 \%$ of the distant LIRGs are located within the solid rectangle in Fig. 7, hence at the location of galaxies experiencing a starburst in the simulation. The remaining $25 \%$ of the distant LIRGs lie in the dashed rectangle that includes the region of post-starbursts but also galaxies with successive or longer starbursts. Hence, the results of the simulation from SIM1 simulation show that the observed galaxies are mainly coherent with being starbursting galaxies.

As a second step, we designed a new simulation, with $80 \%$ of the galaxies experiencing an ongoing starburst (SIM2), to sample the locus of the distant LIRGs better and therefore better study the properties of the starbursts themselves. A third and last simulation (SIM3) was generated to quantify the probability that the distant LIRGs experienced a previous starburst during the past 2 Gyear. Half of the galaxies in SIM3, i.e. 100000 galaxies, have experienced a previous starburst during the last 2 Gyear, which ended before the onset of the ongoing starburst.

The individual positions of the distant LIRGs are compared to the SIM2 realizations in Figs. 8 and 9 in the H8-D4000 and $\mathrm{H} \delta_{\mathrm{A}}$-D4000 diagrams, respectively. UDSR09, which is lying at the bottom of the simulation, is a clear outlier. Liang et al. (2005) studied this object carefully and its optical spectrum shows strong metal absorption lines, such as $\mathrm{Ca} \mathrm{H} \mathrm{K}$, G-band, $\mathrm{Mg} \mathrm{H}, \mathrm{Na} \mathrm{D}$ lines, but weak Balmer absorption lines. The X-ray emission of this object, in addition, is mainly associated to an AGN. As a consequence, an AGN can partially contribute to the MIR luminosity. Note that the observed galaxies are not identical in both figures neither in numbers nor in identity because 


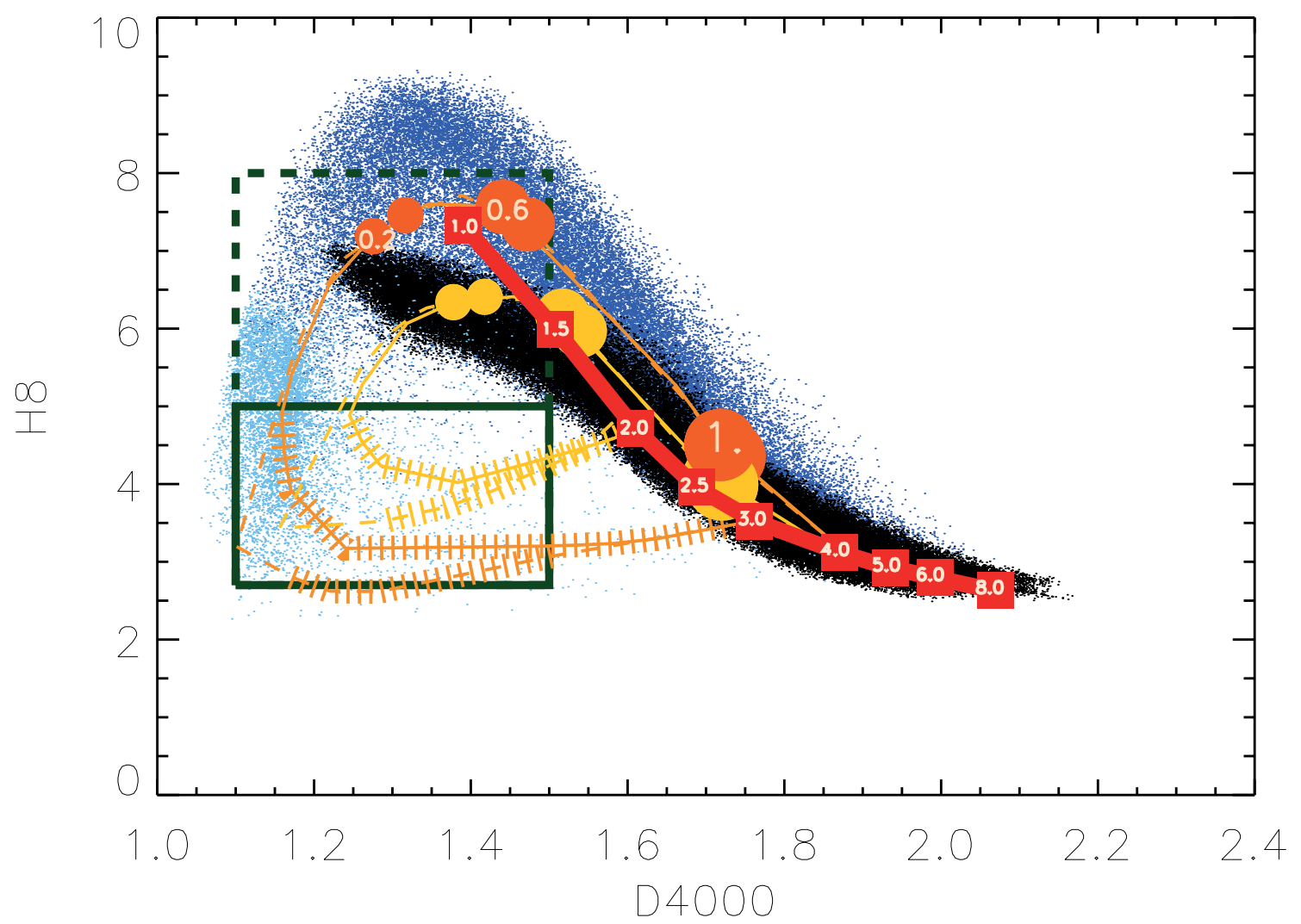

Fig. 7. Location of the 200000 Monte Carlo realizations using the parameters of SIM1 (see Table 5) in the H8 versus $4000 \AA$ (D4000) break diagram. Light blue points (lower-left): starbursting galaxies. Dark blue points (upper part): post-starburst galaxies (galaxies having experienced a recent starburst which ended less than 2 Gyear ago). Black points: galaxies with continuous star formation in the two past Gyears. Bold red line: track followed by an individual galaxy with continuous star formation (numbers in squares $=$ age in Gyear) generated with GALAXEV $(\gamma=2$., $\mu=0.3, \tau_{\mathrm{V}}=3.0, V_{\text {disp }}=200 \mathrm{~km} \mathrm{~s}^{-1}$ ). Orange loops: effect of starbursts of $5 \times 10^{7}$ (dashed line) and $10^{8}$ (plain line) years starting after 2 Gyear (light orange) and 3 Gyear (dark orange) of continuous star formation. The line is hatched during the starburst phase. The size of the orange dots is proportional to the time counted in $10^{8}$ years units after the beginning of the burst. The dark rectangle with dashed contours: area where the distant LIRGS are located. The dark rectangle with a solid line: area where $75 \%$ of the sample lie (see Fig. 8 for the position of the individual galaxies with error bars). Triangles: galaxies corrected for the underlying nebular emission line in $\mathrm{H} 8$ as derived from the observed lower-order Balmer lines corrected for attenuation from the Balmer lines ratio. Stars: galaxies with negligible emission Balmer line in emission, hence uncorrected for $\mathrm{H} 8$ emission. Squares: corrected for the underlying H8 emission line using only one Balmer emission line available with dust attenuation computed from the IR (from the ratio of $S F R(\mathrm{IR})$ over $S F R(\mathrm{H} \beta)$ ). Circles: no correction for the underlying potential emission line because no information is available on the Balmer emission lines.

both $\mathrm{H} 8$ and $\mathrm{H} \delta_{\mathrm{A}}$ cannot both be measured for all individual galaxies. The dots correspond to the same simulated galaxies in both figures and in both cases. Note that the observed LIRGs do lie below the continuous star formation regime in both figures, although in slightly different locations. These differences will be discussed in Sect. 6.5 when we will present the resulting PDFs. Note also that the distant LIRGs are distributed in two populations in Fig. 8, one located at the bottom of the diagram and a second in the upper-left. The second population corresponds to simulated galaxies that experienced a succession of two starbursts during the past 2 Gyears. Such histories are expected in the framework of major mergers of spiral galaxies with several encounters between two galaxies.

\subsection{Determination of the Scalo parameter, $S F R /\langle S F R\rangle$}

The PDF obtained for the Scalo parameter of a typical galaxy with $S F R /\langle S F R\rangle \sim 2.7_{-1.0}^{+7.3}(1-\sigma)$ is represented in Fig. 10a. In about $60 \%$ of the distant LIRGs, the PDF converge towards a determination of the Scalo parameter. For 10 out of 17 galaxies for $\mathrm{H} \delta_{\mathrm{A}}$ (see Table 6), we compute a median value of $S F R /\langle S F R\rangle=3.0_{-0.6}^{+3.5}(1-\sigma)$. For 12 out of 22 for $\mathrm{H} 8$ (see
Table 7) we find $S F R /\langle S F R\rangle=4_{-1}^{+1}(1-\sigma)$. These two results are consistent with each other, as illustrated by Figs. 11a and $\mathrm{c}$. The fact that the H8-D4000 diagram provides the tightest constraint on the Scalo parameter is due to the fact that the sky background is lower in the bluer wavelength range of $\mathrm{H} 8$ than in $\mathrm{H} \delta_{\mathrm{A}}$. We therefore use that range of values for the Scalo parameter in the next set of computations. The fact that the LIRGs are found to produce stars at a rate that is four times higher than their averaged past $S F R$ confirms that they are experiencing a major phase of star formation in their lifetime. This result is consistent with the large $L_{\mathrm{IR}}$ and $S F R(\mathrm{IR})$.

As noted above, the H8-D4000 diagram provides the tightest constraint on the Scalo parameter with $S F R /\langle S F R\rangle=4_{-0.9}^{+1.0}$. Combined with the ongoing $S F R$ measured from the MIR emission for the distant LIRGs (quoted in Table 1) for individual galaxies and with a median value of: $\overline{S F R}=52_{-33}^{+34} M_{\odot} \mathrm{yr}^{-1}$, the Scalo parameter allows us to compute the averaged past $S F R$ of the distant LIRGs: $\langle S F R\rangle \sim 13 \pm 3 M_{\odot} \mathrm{yr}^{-1}$.

If we assume that the progenitors of the distant LIRGs formed stars at a constant rate equal to the averaged past $S F R$, a median stellar mass of $7 \times 10^{10} M_{\odot}$ (see Sect. 4) is assembled in 


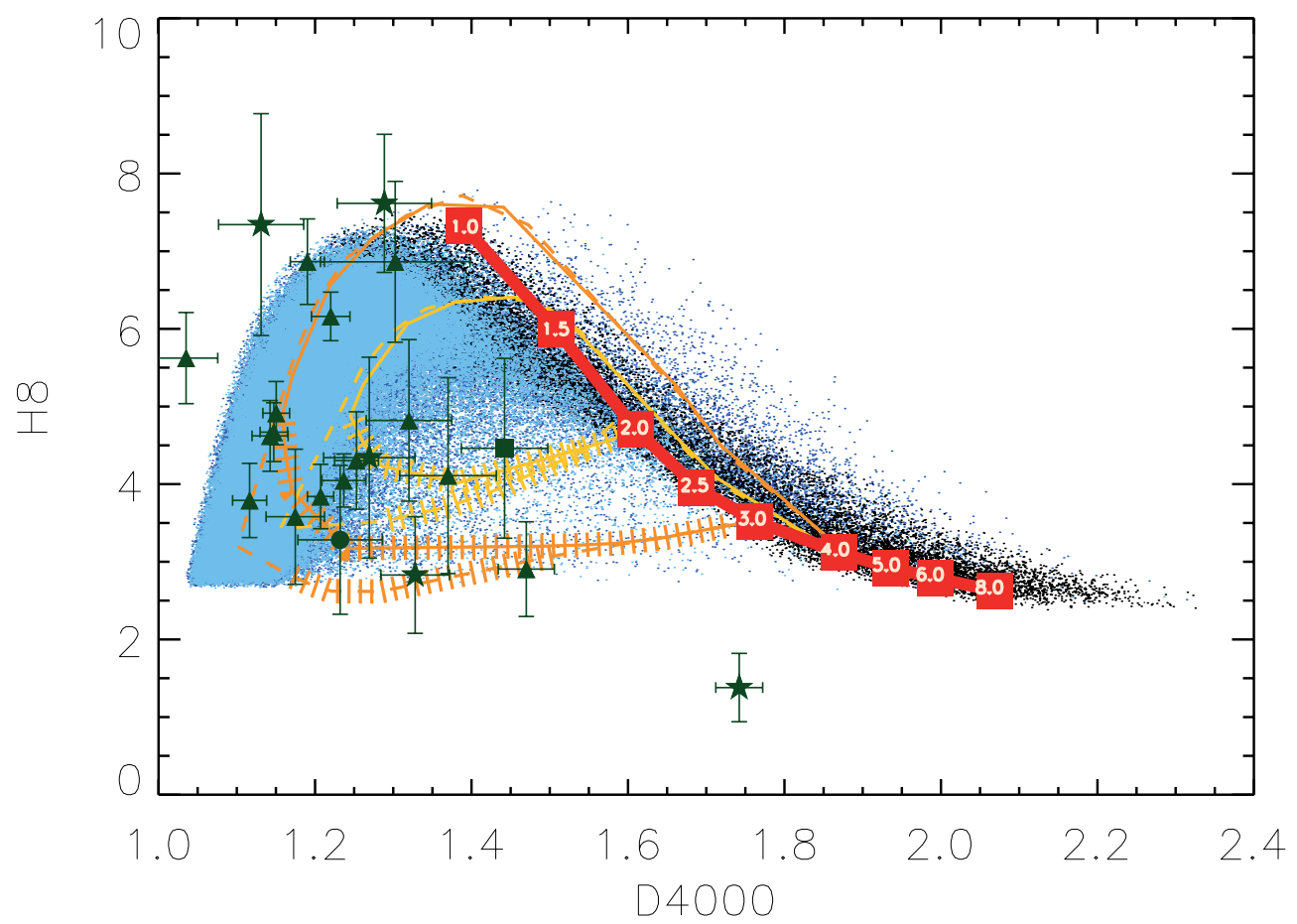

Fig. 8. Same as in Fig. 7 for the SIM2 Monte Carlo simulation of 200000 model galaxies. The distant LIRGs are represented with green points with error bars.

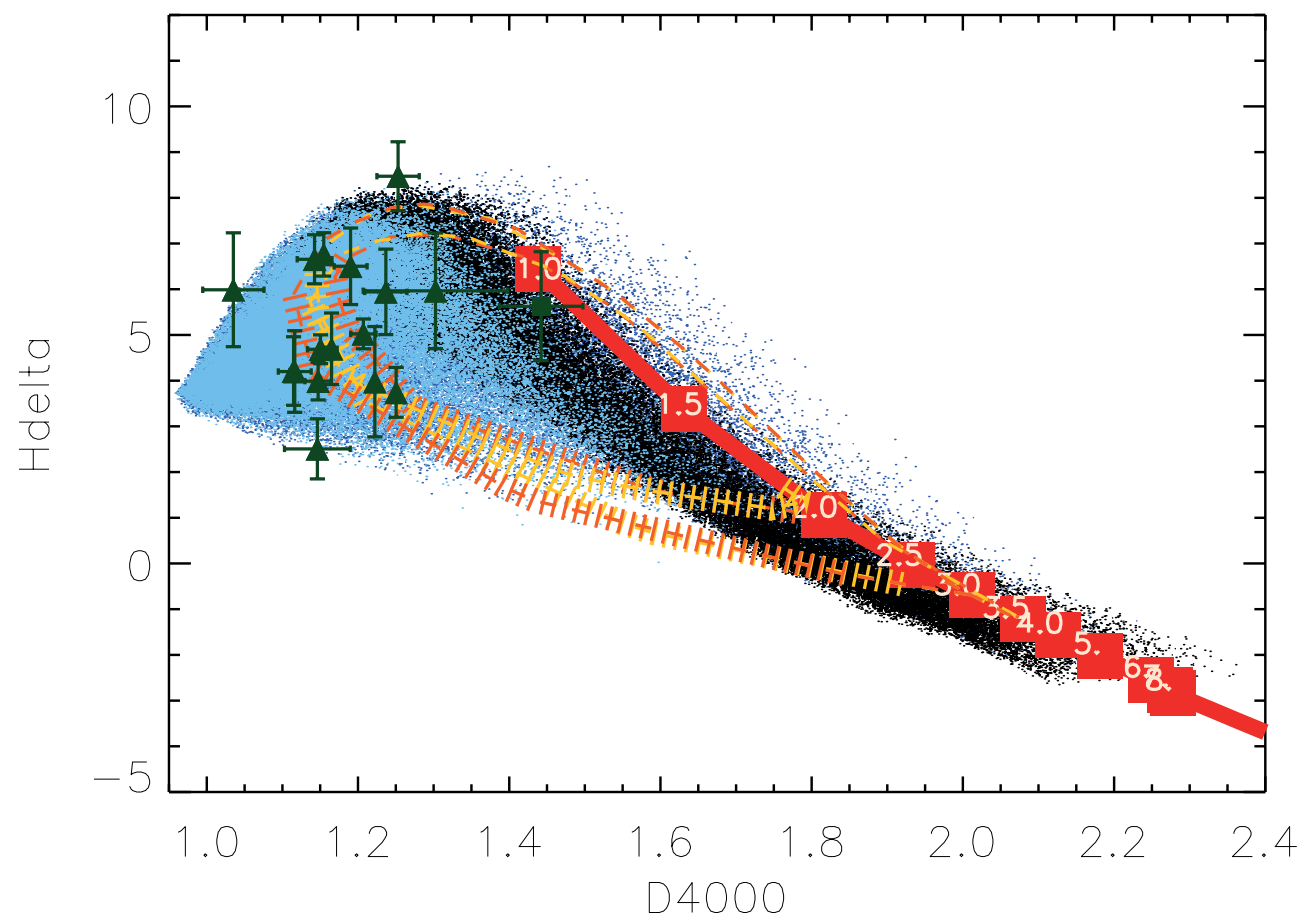

Fig. 9. Location of the 200000 Monte Carlo realizations using the parameters of SIM2 (see Table 5) in the H $\delta_{\mathrm{A}}$ versus $4000 \AA$ (D4000) break diagram. Light blue points (lower-left): starbursting galaxies. Dark blue points (upper part): post-starburst galaxies (galaxies having experienced a recent starburst that ended less than 2 Gyear ago). Black points: galaxies with continuous star formation in the two past Gyears. Bold red line: track followed by an individual galaxy with continuous star formation (numbers in squares $=$ age in Gyear) generated with GALAXEV $(\gamma=4$., $\mu=0.3, \tau_{\mathrm{V}}=3.0, V_{\text {disp }}=200 \mathrm{~km} \mathrm{~s}^{-1}$ ). Orange loops: effect of starbursts of $5 \times 10^{7}$ (dashed line) and $10^{8}$ (plain line) years starting after 2 Gyear (light orange) and 3 Gyear (dark orange) of continuous star formation. The line is hatched during the starburst phase. The size of the orange dots is proportional to the time counted in $10^{8}$ years units after the beginning of the burst. Triangles: galaxies corrected for the underlying nebular emission line in $\mathrm{H} \delta_{\mathrm{A}}$ as derived from the observed lower order Balmer lines corrected for attenuation from the Balmer lines ratio. Stars: galaxies with a negligible emission Balmer line in emission, hence uncorrected for $\mathrm{H} \delta_{\mathrm{A}}$ emission. Squares: corrected for the underlying $\mathrm{H} \delta_{\mathrm{A}}$ emission line using only one Balmer emission line available with dust attenuation computed from the IR (from the ratio of $S F R(\operatorname{IR})$ over $S F R(\mathrm{H} \beta)$ ). Circles: no correction for the underlying potential emission line because no information is available on the Balmer emission lines. 

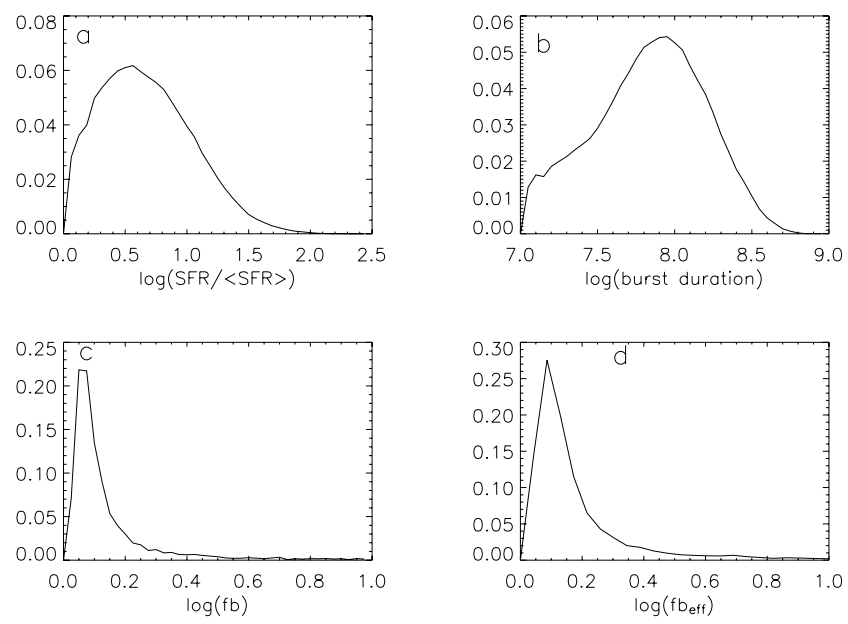

Fig. 10. Example of PDF obtained for 1 galaxy: a) the Scalo parameter (Sect. 6.2); b) burst duration (Sect. 6.2); c) burst fraction (Sect. 6.2); d) effective burst fraction (Sect. 6.2).

about 5.5 \pm 1.5 Gyear (light-weighted age), implying that the first dominant stellar populations formed at a redshift $z \sim 4.5 \pm 1.5$.

\subsection{Determination of the burst duration}

In the $\mathrm{H} \delta_{\mathrm{A}}$-D4000 diagram, the burst duration, $\tau_{\mathrm{B}}$, can be constrained for 11 galaxies (Table 6) for which we obtain a median of $\tau_{\mathrm{B}}=1.3_{-0.5}^{+1.9} \times 10^{8}$ years, e.g. Fig. 10b. Using H8, the technique converges for 14 galaxies to $\tau_{\mathrm{B}}=1.0_{-0.6}^{+1.6} \times 10^{8}$ years. Here again, as for the Scalo parameter in the previous section, we find a consistent result using both $\mathrm{H} 8$ and $\mathrm{H} \delta_{\mathrm{A}}$. Both indicate that the bursts are short-lived over about 0.1 Gyear. The large dispersion between the various galaxies may result from different initial gas mass fractions or triggering mechanisms for the burst.

\subsection{Determination of the burst stellar mass fraction}

The simulations converge to a value of $f_{\mathrm{B}}$ for only $6 / 22$ galaxies in the H8-D4000 diagram and 10/17 in the $\mathrm{H} \delta_{\mathrm{A}}$-D4000 (see the example of UDSR 23 in Fig. 10c). The difficulty of determining the burst stellar mass fraction is not surprising. It results from the fact that as soon as the young stellar population starts to dominate the spectrum of a galaxy, it becomes nearly impossible to measure the fraction of the light due to the underlying older population with good precision. Indeed, the PDFs converge only for galaxies for which the fraction of young stars remains low (see Tables 6, 7).

However, we can still derive the effective burst stellar mass fraction, $f_{\mathrm{B}}^{\text {eff, }}$, which is equal to the mass of stars produced during the burst, i.e. $\tau_{\mathrm{B}} \times S F R$, divided by the total stellar mass of the galaxy, i.e. $t_{\text {form }} \times\langle S F R\rangle$. Since $\tau_{\mathrm{B}}=1.0_{-0.6}^{+1.6} \times 10^{8}$ years and $S F R=52_{-33}^{+34} M_{\odot} \mathrm{yr}^{-1}$, we obtain an effective burst stellar mass fraction of $f_{\mathrm{B}}^{\text {eff }}=10 \pm 9 \%$. Note that a burst producing $10 \%$ of a $7 \times 10^{10} M_{\odot}$ galaxy, will convert $7 \times 10^{9} M_{\odot}$ of molecular gas into stars, which is consistent with the mass of molecular gas observed in local LIRGs and ULIRGs (Sanders \& Mirabel 1996).

\subsection{Testing the "multiple burst" scenario}

A sub-sample of the distant LIRGs lies in the upper-left part of the $\mathrm{H} 8$ and $\mathrm{H} \delta_{\mathrm{A}}$ versus $\mathrm{D} 4000$ diagrams (Figs. 8, 9). This region is populated by Monte Carlo realizations of galaxies for which the ongoing burst of star formation was superimposed on a previous burst that ended less than 2 Gyear ago. In order to quantify the probability of such an occurrence, we generated a third simulation, SIM3, in which half of the realizations experienced two successive bursts during the Past 2 Gyear. Using the same Bayesian statistics, we then computed for each galaxy a probability for various possible durations between the two bursts. Two representative numbers are assigned for each galaxy in Tables 8 and 9: $t_{20}$ and $t_{50}$. These values correspond to lookback times associated with a 20 and a $50 \%$ chance of finding a previous burst that ended $t_{20}$ and $t_{50}$ Gyear before the onset of the ongoing one. For example, in the case of UDSR20, we obtain $t_{20}=0.4$ Gyear and $t_{50}=1$ Gyear from the H8-D4000 diagram (see Table 8), which implies that there is a $50 \%$ chance that a previous starburst occurred 1 Gyear before the onset of the present one and a $20 \%$ chance that the delay was only a 0.4 Gyear. Hence UDSR20 is a good candidate for two successive bursts. We did not consider higher probabilities or longer timescales because of the limited constraints that we can set on those parameters and because after about 1.5 Gyear, the memory of the previous burst is lost with this technique.

Local LIRGs and ULIRGs are known to be predominantly triggered by major mergers (Borne et al. 1999; Sanders \& Mirabel 1996), and numerical simulations of such mergers predict that the two galaxies cross each other several times, potentially inducing a series of bursts separated by a few tens of million years (Mihos \& Hernquist 1996). However, in distant LIRGs, the probability that a previous starburst occurred less than 0.5 Gyear ago is nearly always lower than $20 \%$. This result must be considered together with recent evaluations of the morphological properties of distant LIRGs, which also suggest that most of them are not produced in major mergers (Bell et al. 2005; Zheng et al. 2004; Elbaz \& Cesarsky 2004). Bell et al. (2005) suggest that distant LIRGs could either be non-triggered phases in isolated spirals with larger gas masses, possibly experiencing some infall, or minor mergers, where the dwarf galaxy responsible is not detected. Our determination of a burst duration of 0.1 Gyear and a Scalo parameter of 4, seems to rule out the possibility that distant LIRGs are isolated spirals forming stars at a constant rate over a long duration. The starbursts may instead be triggered by tidal effects and minor mergers in regions of the universe where the local density of galaxies is enhanced, as suggested by Elbaz \& Cesarsky (2003) or by the infall of intergalactic gas (Combes 2005). Further kinematical studies of distant galaxies (see Flores et al. 2006; Puech et al. 2006) will help to distinguish between the various scenarios (mergers, gas infall) discussed here.

\section{Discussion and conclusions}

We have analysed the star formation history of a sample of 25 distant LIRGs $(\bar{z}=0.7)$ that we derived from their stellar spectra (Balmer absorption lines and $4000 \AA$ break). The high-order Balmer absorption line $\mathrm{H} 8$ and the $\mathrm{H} \delta_{\mathrm{A}}$ line provide consistent results although $\mathrm{H} 8$ is adapted more to distant galaxies being located in a bluer region of the spectrum, which is less affected by sky emission lines and which is measured for more distant objects than $\mathrm{H} \delta_{\mathrm{A}}$. Variations at the $30 \%$ level between the burst parameters obtained using one or the other indicator suggest that some effects, such as abundance ratios, which might affect the pseudo-continua surrounding the $\mathrm{H} \delta_{\mathrm{A}}$ line, should be taken into account to improve the models (see Thomas et al. 2004; Korn et al. 2005). 
Table 6. Results concerning the burst duration, the Scalo ratio $S F R /\langle S F R\rangle$, and the burst fraction $\left(f_{\mathrm{B}}\right)$ when using the $\mathrm{H} \delta_{\mathrm{A}}$ data.

\begin{tabular}{|c|c|c|c|c|}
\hline id & $\begin{array}{l}S F R /\langle S F R\rangle \\
{[68 \%]}\end{array}$ & $\begin{array}{l}\left(\tau_{\mathrm{B}}\right)\left[\left(\tau_{\mathrm{B}}^{-}\right),\left(\tau_{\mathrm{B}}^{+}\right)\right] \\
\times 0.1 \text { Gyear [68\%] }\end{array}$ & $\begin{array}{l}f_{\mathrm{B}}\left[f_{\mathrm{B}}^{-}, f_{\mathrm{B}}^{+}\right] \\
(\%)[68 \%]\end{array}$ & $\begin{array}{l}f_{\mathrm{B}}^{\mathrm{e} f f}\left[f_{\mathrm{B}}^{\mathrm{e} f f}, f_{\mathrm{B}}^{\mathrm{eff}+}\right] \\
(\%)[68 \%] \\
(\%)[6]\end{array}$ \\
\hline UDSF07 & $6[3,24]$ & - & $4[3,17]$ & $5[3,25]$ \\
\hline UDSF16 & - & $0.8[0.3,3.0]$ & $3[2,5]$ & $4[3,7]$ \\
\hline UDSF17 & $2[1,5]$ & $4[1.5,6]$ & - & - \\
\hline UDSF18 & $2[1,5]$ & $1[0.5,3]$ & $4[3,13]$ & $5[3,17]$ \\
\hline UDSF19 & - & $1.6[0.4,4.0]$ & $2[1,23]$ & $3[2,10]$ \\
\hline UDSF31 & - & - & - & - \\
\hline UDSR08 & $6[3,27]$ & $0.4[0.2,1.3]$ & $3[2,16]$ & $5[3,26]$ \\
\hline UDSR10 & - & - & - & - \\
\hline UDSR14 & $4[2,13]$ & $0.3[0.1,0.8]$ & $4[2,9]$ & $5[3,14]$ \\
\hline UDSR 20 & - & $5[1,8]$ & - & - \\
\hline UDSR23 & $3[1,10]$ & $0.8[0.3,1.6]$ & $6[4,9]$ & $7[5,19]$ \\
\hline CFRS02 & - & - & - & - \\
\hline CFRS06 & $2[1,4]$ & 2. $[1,4]$ & $2[1,9]$ & $7[5,19]$ \\
\hline CFRS08 & - & - & - & - \\
\hline CFRS10 & $10[5,36]$ & $1[0.2,2.0]$ & $13[7,30]$ & $14[7,47]$ \\
\hline CFRS11 & $3[2,15]$ & - & $3[2,5]$ & $4[3,9]$ \\
\hline CFRS14 & $3[2,6]$ & $3[1,6]$ & - & - \\
\hline
\end{tabular}

Table 7. Results concerning the burst duration, the Scalo ratio $S F R /\langle S F R\rangle$, and the burst fraction $\left(f_{\mathrm{B}}\right)$ when using the H8 data.

\begin{tabular}{|c|c|c|c|c|}
\hline id & $\begin{array}{l}S F R /\langle S F R\rangle \\
(\%)[68 \%]\end{array}$ & $\begin{array}{l}\tau_{\mathrm{B}}\left[\tau_{\mathrm{B}}^{-}, \tau_{\mathrm{B}}^{+}\right] \\
\times 0.1 \text { Gyear [68\%] }\end{array}$ & $\begin{array}{l}f_{\mathrm{B}}\left[f_{\mathrm{B}}^{-}, f_{\mathrm{B}}^{+}\right] \\
(\%)[68 \%]\end{array}$ & $\begin{array}{l}f_{\mathrm{B}}^{\mathrm{e} f f}\left[f_{\mathrm{B}}^{\mathrm{eff}-}, f_{\mathrm{B}}^{\mathrm{eff}+}\right] \\
(\%)[68 \%]\end{array}$ \\
\hline UDSF06 & - & $2[1,6]$ & $3[2,19]$ & $3[2,22]$ \\
\hline UDSF07 & $4[2,13]$ & $0.2[0.1,0.9]$ & - & - \\
\hline UDSF12 & - & $\geq 8.9$ & - & - \\
\hline UDSF17 & - & $5[1,8]$ & - & - \\
\hline UDSF19 & - & - & $2[1,15]$ & $3[2,19]$ \\
\hline UDSF28 & $4[2,8]$ & - & - & - \\
\hline UDSF29b & - & - & - & - \\
\hline UDSF20 & $2[1,5]$ & - & - & - \\
\hline UDSF31 & - & - & - & - \\
\hline UDSR08 & $7[3,30]$ & $0.4[0.2,0.7]$ & - & - \\
\hline UDSR09 & - & $0.4[0.2,0.9]$ & - & - \\
\hline UDSR10 & - & $1[0.3,2.2]$ & $3[2,13]$ & $3[3,15]$ \\
\hline UDSR14 & $5[2,12]$ & $0.8[0.3,1.7]$ & - & - \\
\hline UDSR20 & - & $7[3,8]$ & - & - \\
\hline UDSR 23 & $3[2,7]$ & $1[1,3]$ & - & - \\
\hline CFRS02 & - & $\geq 3.1$ & - & - \\
\hline CFRS06 & $3[1,7]$ & $0.2[0.1,0.7]$ & $4[3,13]$ & $4[3,16]$ \\
\hline CFRS08 & $4[2,10]$ & $0.4[0.1,1.0]$ & $3[2,11]$ & $4[3,14]$ \\
\hline CFRS10 & $8[5,14]$ & $1.0[0.6,2.0]$ & - & - \\
\hline CFRS14 & $4[2,11]$ & $0.6[0.3,1.6]$ & - & - \\
\hline CFRS16 & $7[3,23]$ & $\leq 7.85$ & $4[2,18]$ & $5[3,21]$ \\
\hline CFRS29 & $3[2,9]$ & $2.5[1,5]$ & - & - \\
\hline
\end{tabular}

Comparison of distant LIRGs, selected from ISOCAM and MIPS onboard ISO and Spitzer, to local LIRGs, selected from IRAS and the SDSS, shows that both populations present similar spectral features and therefore suggests that they are experiencing comparable starburst phases. Half of the local LIRGs present D4000 values that are higher than the maximum D4000 of distant LIRGs indicates that the dominant non bursting stellar population is younger for distant LIRGs, as expected.

The first important result of this study is the identification of an optical signature for the presence of a starburst in these galaxies, in spite of their large dust attenuation. While continuous star formation follows a line along decreasing Balmer EW and increasing D4000, a burst superimposed on this population produces a loop that first decreases D4000 and then increases the Balmer line EW. However, after about 1.5 Gyear, the memory of the burst is lost and the galaxy behaves like others that did not experience a starburst. As result, we are limited to study only the averaged past star formation history for lookback times shorter than 1.5 Gyear. The burst characteristics were derived from probability distribution functions (PDF) using a Bayesian statistics as in Kauffmann et al. (2003).

The median ratio of present over averaged $S F R$, the so-called Scalo parameter, for the distant LIRGs is $S F R /\langle S F R\rangle=4 \pm 1$ (we used the H8 line for which a larger sample of galaxies is available and the PDFs present a sharper peak), which indicates that these galaxies are experiencing an atypically intense phase of star formation in their lifetime. A median SFR of $52_{-33}^{+34} M_{\odot} \mathrm{yr}^{-1}$ for the ongoing starbursts was derived from their MIR luminosities; Hence, their mean $S F R$ averaged over their lifetime is $\langle S F R\rangle=13 \pm 3 M_{\odot} \mathrm{yr}^{-1}$. Knowing the median stellar mass for LIRGs of equivalent luminosity and redshift range (from Franceschini et al. 2003), we derived an age for those distant LIRGs of $t_{\text {form }}=5.5 \pm 1.5$ Gyear, suggesting that they formed at $z_{\text {form }}=4.5 \pm 1.5$. 

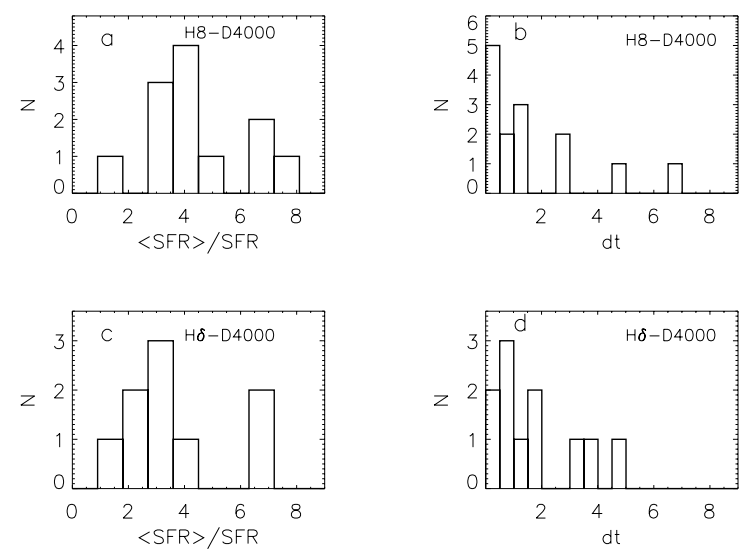

Fig. 11. Distribution of the Scalo parameter and the burst duration; a) the Scalo parameter in the H8-D4000 diagram; b) the burst duration in the H8-D4000 diagram; c) the Scalo parameter in the $\mathrm{H} \delta_{\mathrm{A}}-\mathrm{D} 4000$ diagram; d) the burst duration in the $\mathrm{H} \delta_{\mathrm{A}}-\mathrm{D} 4000$ diagram.

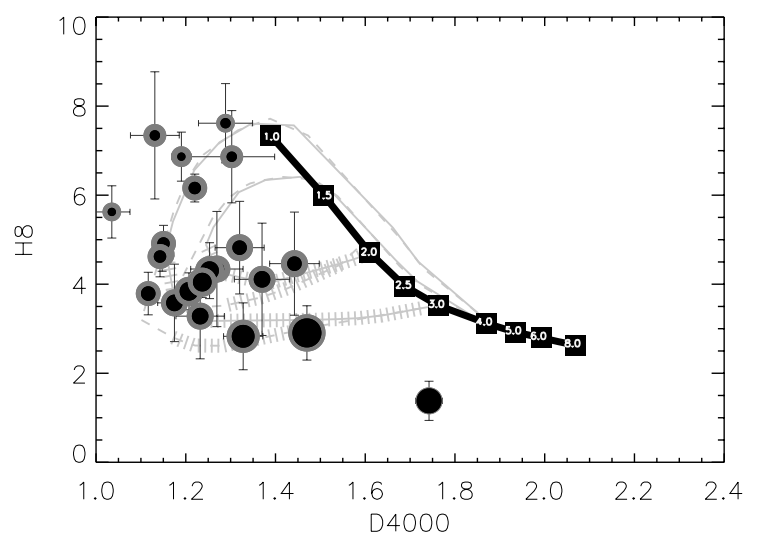

Fig. 12. The lines are the same as in Figs. 7 and 8. The black circles are proportional to $t_{20}$ defined before, while the grey ones to $t_{50}$.

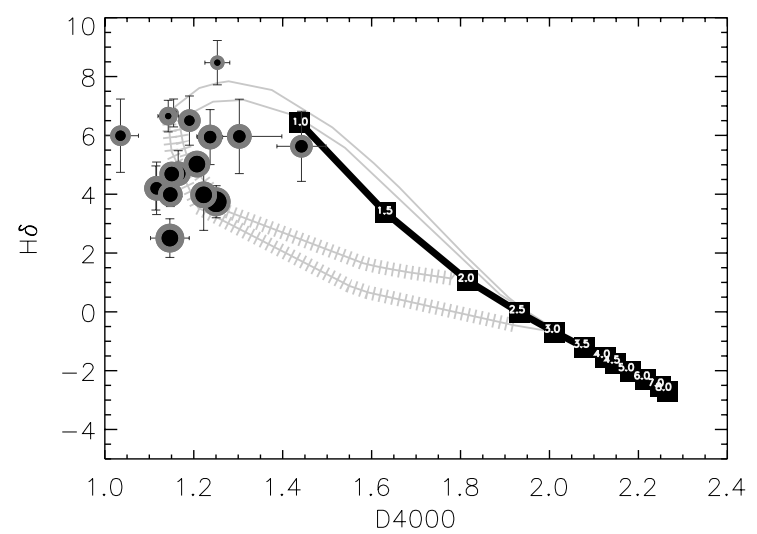

Fig. 13. The lines are the same as in Figs 7 and in 8 . The black circles are proportional to $t_{20}$ defined before, while the grey ones to $t_{50}$.

For the bursts themselves, we computed a median duration of $\tau_{\mathrm{B}}=1.0_{-0.6}^{+1.6} \times 10^{8}$ years, during which the galaxies produced $10 \pm 9 \%$ (the error bar includes $68 \%$ of the galaxy sample) of their stellar mass. This corresponds to a mass of molecular gas of about $7 \times 10^{9} M_{\odot}$, which is consistent with that observed in local LIRGs and ULIRGs (see Sanders \& Mirabel 1996).

We note that all simulations produced in this paper assume the same fixed IMF for both the underlying star formation and the burst of star formation. Some evidence that the formation of low-mass may be less efficient in the environment of active
Table 8. Values of $t_{20}$ (black circle) and $t_{50}$ (grey circle) obtained for the 22 galaxies in the H8-D4000 diagram, to quantify the probability of two successive starbursts (see text).

\begin{tabular}{l|l|l}
\hline \hline id & $\begin{array}{l}t_{20} \\
10^{8} \text { years } \\
(2)\end{array}$ & $\begin{array}{l}t_{50} \\
10^{8} \text { years } \\
(3)\end{array}$ \\
\hline 6.udsf & 7.0 & 13.0 \\
7.udsf & 8.0 & 13.0 \\
12.udsf & 5.0 & 9.0 \\
17.udsf & 6.0 & 12.0 \\
19.udsf & 7.0 & 13.0 \\
28.udsf & 14.0 & 18.0 \\
29.udsf & 8.0 & 13.0 \\
20.udsf & 11.0 & 16.0 \\
31.udsf & 7.0 & 13.0 \\
\hline 8.udsr & 7.0 & 12.0 \\
10.udsr & 9.0 & 14.0 \\
14.udsr & 7.0 & 12.0 \\
20.udsr & 4.0 & 10.0 \\
23.udsr & 6.0 & 12.0 \\
9.udsr & 12.0 & 13.0 \\
\hline 2.cfrs & 5.0 & 11.0 \\
6.cfrs & 9.0 & 14.0 \\
8.cfrs & 9.0 & 14.0 \\
10.cfrs & 4.0 & 9.0 \\
14.cfrs & 6.0 & 12.0 \\
16.cfrs & 8.0 & 13.0 \\
29.cfrs & 5.0 & 11.0 \\
\hline
\end{tabular}

Table 9. Values of $t_{20}$ (black circle) and $t_{50}$ (grey circle) obtained for the 17 galaxies in the $\mathrm{H} \delta_{\mathrm{A}}-\mathrm{D} 4000$ diagram, to quantify the probability of two successive starbursts (see text).

\begin{tabular}{|c|c|c|}
\hline (1) & $\begin{array}{l}t_{20} \\
10^{8} \text { years } \\
\text { (2) }\end{array}$ & $\begin{array}{l}t_{50} \\
10^{8} \text { years } \\
\text { (3) }\end{array}$ \\
\hline 7.udsf & 6.0 & 11.0 \\
\hline 16.udsf & 10.0 & 14.0 \\
\hline 17.udsf & 3.0 & 5.0 \\
\hline 18.udsf & 7.0 & 12.0 \\
\hline 19.udsf & 8.0 & 13.0 \\
\hline 31.udsf & 6.0 & 11.0 \\
\hline 8.udsr & 6.0 & 12.0 \\
\hline 10.udsr & 3.0 & 7.0 \\
\hline 14.udsr & 7.0 & 12.0 \\
\hline 20.udsr & 5.0 & 11.0 \\
\hline 23.udsr & 7.0 & 12.0 \\
\hline 2.cfrs & 6.0 & 12.0 \\
\hline 6.cfrs & 8.0 & 13.0 \\
\hline 8.cfrs & 6.0 & 12.0 \\
\hline 10.cfrs & 5.0 & 10.0 \\
\hline 11.cfrs & 8.0 & 14.0 \\
\hline 14.cfrs & 3.0 & 9.0 \\
\hline
\end{tabular}

star formation in the solar neighborhood were suggested in the past (Larson 1986; Scalo 1986; Maeder 1993). A top-heavy IMF could also account for the enhanced ratio of light elements to iron in massive early-type galaxies (Worthey et al. 1992) and for the relative enrichment of oxygen to iron in the intra-cluster medium (Arnaud et al. 1992). In our study, the occurrence of a top-heavy IMF in the burst episode would only weakly influence the derived burst duration timescales, which are set by the spectral signature of massive A to F stars. However, a top-heavy IMF during the burst phase would imply a much lower contribution to the total galaxy mass by longer-lived, low-mass stars. 
Finally, we discussed the possibility that the distant LIRGs had experienced a previous starburst prior to the ongoing one during the past 1.5 Gyear. While most galaxies are not consistent with a merger scenario where two galaxies merge in several phases producing a series of bursts separated by a few ten million years (Mihos \& Hernquist 1996), the majority present more than $50 \%$ chance of having experienced a previous burst in the past 1.5 Gyear, i.e. since $z \sim 1$. If these properties are typical of LIRGs between $z=1$ and $z=0$, then this suggests that the population of galaxies experiencing LIRG phases experienced on average 2 to 3 LIRG phases since $z=1$ and up to 4 since their birth around $z=4-5$, as also suggested by Hammer et al. (2005). This scenario is not consistent with the formation of distant LIRGs through the continuous star formation characterizing isolated spiral galaxies as has been argued independently based on their morphology. Instead, minor mergers, tidal interactions, and gas accretion all remain equally plausible triggering mechanisms for more than half of the distant LIRGs that do not harbor the morphology of major mergers.

Acknowledgements. We wish to thank the anonymous referee for constructive remarks that helped improving the paper, in particular on the wavelet analysis. We also wish to thank Anna Gallazzi, Nicolas Gruel, Emmanuel Moy, and Jean Luc Starck for helpful discussions and comments, and Emeric Le Floc'h for technical support with the MIPS data. S.C. thanks the Alexander von Humboldt Foundation, the Federal Ministry of Education and Research, and the Programe for Investment in the Future (ZIP) of the German Government for their support.

\section{References}

Arnaud, M., Rothenflug, R., Boulade, O., Vigroux, L., \& Vangioni-Flam, E. 1992, A\&A, 254, 49

Balogh, M. L., Morris, S. L., Yee, H. K. C., Carlberg, R. G., \& Ellingson, E. 1999, ApJ, 527, 54

Bell, E. F., Papovich, C., Wolf, C., et al. 2005, ApJ, 625, 23

Bica, E., \& Alloin, D. 1986, A\&A, 162, 21

Blain, A. W., Kneib, J.-P., Ivison, R. J., \& Smail, I. 1999, ApJ, 512, L87

Borne, K. D., Bushouse, H., Colina, L., et al. 1999, Ap\&SS, 266, 137

Bruzual, G., \& Charlot, S. 2003, MNRAS, 344, 1000

Bruzual, A., G. 1983, ApJ, 273, 105

Cardiel, N., Elbaz, D., Schiavon, R. P., et al. 2003, ApJ, 584, 76

Charlot, S., \& Fall, S. M. 2000, ApJ, 539, 718
Chary, R., Casertano, S., Dickinson, M. E., et al. 2004, ApJS, 154, 80 Chary, R., \& Elbaz, D. 2001, ApJ, 556, 562

Combes, F. 2005, in The Evolution of Starbursts, ed. S. Hüttmeister, E. Manthey, D. Bomans, \& K. Weis, AIP Conf. Proc., 783, 43

Dale, D. A., \& Helou, G. 2002, ApJ, 576, 159

Elbaz, D., Cesarsky, C. J., Chanial, P., et al. 2002, A\&A, 384, 848

Elbaz, D., Le Floc'h, E., Dole, H., \& Marcillac, D. 2005, A\&A, 434, L1

Fadda, D., Flores, H., Hasinger, G., et al. 2002, A\&A, 383, 838

Flores, H., Hammer, F., Elbaz, D., et al. 2004, A\&A, 415, 885

Flores, H., Hammer, F., Puech, M., Amram, P., \& Balkowski, C. 2006, A\&A, accepted

Franceschini, A., Berta, S., Rigopoulou, D., et al. 2003, A\&A, 403, 501

Gorgas, J., Cardiel, N., Pedraz, S., \& González, J. J. 1999, A\&AS, 139, 29

Hammer, F., Flores, H., Elbaz, D., et al. 2005, A\&A, 430, 115

Hopkins, A. M., Miller, C. J., Nichol, R. C., et al. 2003, ApJ, 599, 971

Kauffmann, G., Heckman, T. M., White, S. D. M., et al. 2003, MNRAS, 341, 33

Kim, D.-C., \& Sanders, D. B. 1998, ApJS, 119, 41

Korn, A. J., Maraston, C., \& Thomas, D. 2005, A\&A, 438, 685

Lagache, G., \& Dole, H. 2001, A\&A, 372, 702

Lagache, G., Puget, J. L., \& Dole, H. 2005, MNRAS, accepted

Larson, R. B. 1986, in Stellar Populations, 101

Le Borgne, J.-F., Bruzual, G., Pelló, R., et al. 2003, A\&A, 402, 433

Le Floc'h, E., Papovich, C., Dole, Bell, E., et al. 2005, ApJS, accepted

Liang, Y. C., Hammer, F., Flores, H., et al. 2004, A\&A, 423, 867

Liang, Y. C., Hammer, F., Flores, H., et al. 2005, Chin. J. Astron. Astrophys., 5, 335

Maeder, A. 1993, in The Feedback of Chemical Evolution on the Stellar Content of Galaxies, 173

Marano, B., Zamorani, G., \& Zitelli, V. 1988, MNRAS, 232, 111

Marcillac, D., Elbaz, D., Chary, R. R., et al. 2005, A\&A, 451, 57

Mihos, J. C., \& Hernquist, L. 1996, ApJ, 464, 641

Osterbrock, D. E. 1989, S\&T, 78, 491

Papovich, C., Dole, H., Egami, E., et al. 2004, ApJS, 154, 70

Pasquali, A., Kauffmann, G., \& Heckman, T. M. 2005, MNRAS, 361, 1121

Poggianti, B. M., \& Barbaro, G. 1997, A\&A, 325, 1025

Puech, M., Flores, H., Hammer, F., \& Lehnert, M. D. 2006, ArXiv Astrophysics e-prints

Puget, J. L., Lagache, G., Clements, D. L., et al. 1999, A\&A, 345, 29

Scalo, J. M. 1986, Fund. Cosmic Phys., 11, 1

Smail, I., Kuntschner, H., Kodama, T., et al. 2001, MNRAS, 323, 839

Soifer, B. T., \& Neugebauer, G. 1991, AJ, 101, 354

Starck, J.-L., \& Murtagh, F. 1994, A\&A, 288, 342

Starck, J. L., Pantin, E., \& Murtagh, F. 2002, PASP, 114, 1051

Stetson, P. B., \& Aikman, C. L. 1987, AJ, 93, 1439

Thomas, D., Maraston, C., \& Korn, A. 2004, MNRAS, 351, L19

Worthey, G., Faber, S. M., \& Gonzalez, J. J. 1992, ApJ, 398, 69

Worthey, G., \& Ottaviani, D. L. 1997, ApJS, 111, 377

Zamorani, G., Mignoli, M., Hasinger, G., et al. 1999, A\&A, 346, 731

Zheng, X. Z., Hammer, F., Flores, H., Assémat, F., \& Pelat, D. 2004, A\&A, 421, 847 